\title{
A Viscosity Hybrid Steepest Descent Method for Equilibrium Problems, Variational Inequality Problems, and Fixed Point Problems of Infinite Family of Strictly Pseudocontractive Mappings and Nonexpansive Semigroup
}

\author{
Haitao Che and Xintian Pan \\ School of Mathematics and Information Science, Weifang University, Weifang, Shandong 261061, China \\ Correspondence should be addressed to Haitao Che; haitaoche@163.com
}

Received 28 May 2013; Accepted 18 June 2013

Academic Editor: Sehie Park

Copyright (C) 2013 H. Che and X. Pan. This is an open access article distributed under the Creative Commons Attribution License, which permits unrestricted use, distribution, and reproduction in any medium, provided the original work is properly cited.

In this paper, modifying the set of variational inequality and extending the nonexpansive mapping of hybrid steepest descent method to nonexpansive semigroups, we introduce a new iterative scheme by using the viscosity hybrid steepest descent method for finding a common element of the set of solutions of a system of equilibrium problems, the set of fixed points of an infinite family of strictly pseudocontractive mappings, the set of solutions of fixed points for nonexpansive semigroups, and the sets of solutions of variational inequality problems with relaxed cocoercive mapping in a real Hilbert space. We prove that the sequence converges strongly to a common element of the above sets under some mild conditions. The results shown in this paper improve and extend the recent ones announced by many others.

\section{Introduction}

Let $H$ be a real Hilbert space with inner product $\langle\cdot, \cdot\rangle$ and induced norm $\|\cdot\|$. Let $C$ be a nonempty closed convex subset of $H$ and let $F: C \times C \rightarrow R$ be a bifunction. We consider the following equilibrium problem (EP) which is to find $x^{*} \in C$ such that

$$
\text { EP: } F\left(x^{*}, y\right) \geq 0, \quad \forall y \in C .
$$

The set of solutions of EP is denoted by $\operatorname{EP}(F)$.

Let $\left\{F_{i}, i=1,2, \ldots, N\right\}$ be a finite family of bifunctions from $C \times C$ into $R$, where $R$ is the set of real numbers. The system of equilibrium problems for $\left\{F_{1}, F_{2}, \ldots, F_{N}\right\}$ is to find a common element $x^{*} \in C$ such that

$$
\begin{gathered}
F_{1}\left(x^{*}, y\right) \geq 0, \quad \forall y \in C, \\
F_{2}\left(x^{*}, y\right) \geq 0, \quad \forall y \in C, \\
\vdots \\
F_{N}\left(x^{*}, y\right) \geq 0, \quad \forall y \in C .
\end{gathered}
$$

We denote the set of solutions of (2) by $\cap_{k=1}^{N} \operatorname{SEP}\left(F_{k}\right)$, where $\operatorname{SEP}\left(F_{k}\right)$ is the set of solutions to the equilibrium problems, that is,

$$
F_{k}\left(x^{*}, y\right) \geq 0, \quad \forall y \in C .
$$

If $N=1$, then the problem (2) is reduced to the equilibrium problems.

If $N=1$ and $F\left(x^{*}, y\right)=\left\langle T x^{*}, y-x^{*}\right\rangle$, then the problem (2) is reduced to the variational inequality problems of finding $x^{*} \in C$ such that

$$
\left\langle T x^{*}, y-x^{*}\right\rangle \geq 0, \quad \forall y \in C .
$$

The set of solutions of (4) is denoted by $\operatorname{VI}(C, T)$.

The equilibrium problem is very general in the sense that it includes, as special cases, fixed point problems, variational inequality problems, optimization problems, Nash equilibrium problems in noncooperative games, and numerous problems in physics, economics, and others. Some methods have been proposed to solve $\operatorname{VI}(C, T), \operatorname{EP}(F)$, and $\operatorname{SEP}\left(F_{k}\right)$; see, for example, [1-29] and references therein. Formulations 
(2) extend this formulism to such problems, covering in particular various forms of feasibility problems [30, 31].

Definition 1. One-parameter family mapping $\Gamma=\{T(t): t \in$ $R^{+}$from $C$ into itself is said to be a nonexpansive semigroup on $\mathrm{C}$ if it satisfies the following conditions:

(i) $T(0) x=x$ for all $x \in C$,

(ii) $T(s+t)=T(s) T(t)$ for all $s, t \in R^{+}$,

(iii) for each $x \in C$, the mapping $T(t) x$ is continuous,

(iv) $\|T(t) x-T(t) y\| \leq\|x-y\|$ for all $x, y \in C$ and $t \in R^{+}$.

Remark 2. We denote by $F(\Gamma)$ the set of all common fixed points of $\Gamma$, that is, $F(\Gamma):=\bigcap_{t \in R^{+}} F(T(t))=\{x \in C: T(t) x=$ $x\}$.

Let $B: C \rightarrow H$ be a nonlinear mapping. Now, we recall the following definitions.

(1) $B$ is said to be monotone if

$$
\langle B x-B y, x-y\rangle \geq 0, \quad \forall x, y \in C .
$$

(2) $B: C \rightarrow C$ is called $\omega$-Lipschitzian if there exists a positive constant $\omega$ such that

$$
\|B x-B y\| \leq \omega\|x-y\|, \quad \forall x, y \in C .
$$

(3) $B$ is said to be $\eta$-strongly monotone if there exists a positive constant $\eta$ such that

$$
\langle B x-B y, x-y\rangle \geq \eta\|x-y\|^{2}, \quad \forall x, y \in C .
$$

(4) $B$ is said to be nonexpansive if

$$
\|B x-B y\| \leq\|x-y\|, \quad \forall x, y \in C .
$$

And $F(B)$ denotes the set of fixed points of the mapping $B$, that is, $F(B)=\{x \in C: B x=x\}$.

(5) $B: C \rightarrow C$ is said to be $k$-strictly pseudocontractive mapping if there exists a constant $0 \leq k<1$ such that

$$
\begin{array}{r}
\|B x-B y\|^{2} \leq\|x-y\|^{2}+k\|(I-B) x-(I-B) y\|^{2}, \\
\forall x, y \in C .
\end{array}
$$

(6) $B$ is said to be $\alpha$-inverse-strongly monotone if there exists a constant $\alpha>0$ such that

$$
\langle B x-B y, x-y\rangle \geq \alpha\|B x-B y\|^{2}, \quad \forall x, y \in C .
$$

(7) $B$ is said to be relaxed $(u, v)$-cocoercive if there exist positive real numbers $u, v$ such that

$$
\begin{aligned}
\langle B x-B y, x-y\rangle \geq & (-u)\|B x-B y\|^{2} \\
& +v\|x-y\|^{2}, \quad \forall x, y \in C .
\end{aligned}
$$

(8) A linear bounded operator $B$ is strong positive if there exists a constant $\bar{\gamma}>0$ with the property

$$
\langle B x, x\rangle \geq \bar{\gamma}\|x\|^{2}, \quad \forall x \in C .
$$

(9) A set-valued mapping $Q: H \rightarrow 2^{H}$ is called monotone if for all $x, y \in H, f \in Q x$ and $g \in Q y$ imply $\langle x-y, f-g\rangle \geq 0$.

(10) A monotone mapping $Q: H \rightarrow 2^{H}$ is called maximal if the graph $G(Q)$ of $Q$ is not properly contained in the graph of any other monotone mapping. It is well known that a monotone mapping $Q$ is maximal if and only if for $(x, f) \in H \times H,\langle x-y, f-g\rangle \geq 0$ for every $(y, g) \in G(Q)$ implies $f \in Q x$.

Iterative methods for nonexpansive mappings have recently been applied to solve convex minimization problems. Convex minimization problems have a great impact and influence on the development of almost all branches of pure and applied sciences. A typical problem is to minimize a quadratic function over the set of the fixed points of a nonexpansive mapping on a real Hilbert space $H$ :

$$
\theta(x)=\frac{1}{2}\langle A x, x\rangle-\langle x, b\rangle, \quad \forall x \in F(S),
$$

where $A$ is a linear bounded operator, $F(S)$ is the fixed point set of a nonexpansive mapping $S$, and $b$ is a given point in $H$ [16].

For finding a common element of the set of fixed points of nonexpansive mappings and the set of the variational inequalities, in 2006, Marino and $\mathrm{Xu}$ [16] introduced the general iterative method and proved that for a given $x_{0} \in H$, the sequence $\left\{x_{n}\right\}$ generated by the algorithm

$$
x_{n+1}=\alpha_{n} \gamma f\left(x_{n}\right)+\left(I-\alpha_{n} B\right) T x_{n},
$$

where $T$ is a self-nonexpansive mapping on $H, f$ is an $\alpha$ contraction of $H$ into itself (i.e., $\|f(x)-f(y)\| \leq \alpha \| x-$ $y \|, \forall x, y \in H$ and $\alpha \in(0,1)),\left\{\alpha_{n}\right\} \subset(0,1)$ satisfies certain conditions, and $B$ is strongly positive bounded linear operator on $H$ and converges strongly to fixed point $x^{*}$ of $T$ which is the unique solution to the following variational inequality:

$$
\left\langle(\gamma f-B) x^{*}, x^{*}-x\right\rangle \leq 0, \quad \forall x \in F(T),
$$

which is the optimality condition for the minimization problem

$$
\min _{x \in F(S) \cap \mathrm{EP}} \frac{1}{2}\langle B x, x\rangle-h(x),
$$

where $h$ is a potential function for $r f$ (i.e., $h^{\prime}(x)=r f(x)$ for $z \in H$ ).

Takahashi and Toyoda [32] introduced the following iterative scheme:

$$
\begin{gathered}
x_{0} \in C, \\
x_{n+1}=\gamma_{n} x_{n}+\left(1-\gamma_{n}\right) \operatorname{SP}_{C}\left(x_{n}-\alpha_{n} B x_{n}\right),
\end{gathered}
$$


where $B$ is a $\xi$-inverse-strongly monotone mapping, $\left\{\gamma_{n}\right\}$ is a sequence in $(0,1)$, and $\left\{\alpha_{n}\right\}$ is a sequence in $(0,2 \xi)$. They showed that if $F(S) \cap \operatorname{VI}(C, B) \neq \emptyset$, then the sequence $\left\{x_{n}\right\}$ generated by (17) converges weakly to some $z \in F(S) \cap$ $\mathrm{VI}(C, B)$.

Yamada [33] introduced the following iterative scheme called the hybrid steepest descent method:

$$
x_{n+1}=S x_{n}+\alpha_{n} \mu B S x_{n}, \quad n \in N \text {, }
$$

where $x_{1}=x \in H,\left\{\alpha_{n}\right\} \subset(0,1)$, and let $B: H \rightarrow H$ be a strongly monotone and Lipschitz continuous mapping and $\mu$ is a positive real number. He proved that the sequence $\left\{x_{n}\right\}$ generated by (18) converges strongly to the unique solution of $F(S) \cap \operatorname{VI}(C, B)$.

Let $C$ be a nonempty closed convex subset of $H$. Given $r>0$ the operators $J_{r}^{F}: H \rightarrow C$ defined by

$$
J_{r}^{F} x=\left\{z \in C: F(z, y)+\frac{1}{r}(y-z, z-x) \geq 0, \forall y \in C\right\}
$$

are called the resolvent of $F$ (see [19]). It is shown in [19] that under suitable hypotheses on $F$ (to be stated precisely in Section 2), $J_{r}^{F}: H \rightarrow C$ is single-valued and firmly nonexpansive and satisfied $F\left(J_{r}^{F}\right)=\mathrm{EP}(F), \forall r>0$.

For finding a common element of $\operatorname{EP}(F) \cap F(S)$, S. Takahashi and W. Takahashi [23] introduced an iterative scheme by the viscosity approximation method for finding a common element of the set of solution (1) and the set of fixed points of a nonexpansive mapping in a Hilbert space. Let $S: C \rightarrow H$ be a nonexpansive mapping. Starting with arbitrary initial point $x_{1} \in H$, define sequences $\left\{x_{n}\right\}$ and $\left\{u_{n}\right\}$ recursively by

$$
\begin{gathered}
F\left(u_{n}, y\right)+\frac{1}{r_{n}}\left\langle y-u_{n}, u_{n}-x_{n}\right\rangle \geq 0, \quad \forall y \in C, \\
x_{n+1}=\alpha_{n} f\left(x_{n}\right)+\left(1-\alpha_{n}\right) S u_{n}, \quad \forall n \in N .
\end{gathered}
$$

They proved that under certain appropriate conditions imposed on $\left\{\alpha_{n}\right\}$ and $\left\{r_{n}\right\}$, the sequences $\left\{x_{n}\right\}$ and $\left\{u_{n}\right\}$ converge strongly to $z \in F(S) \cap \operatorname{EP}(F)$, where $z=$ $P_{F(S) \cap \mathrm{EP}(F)} f(z)$.

In 2012, Chamnarnpan and Kumam [34] introduced the following explicit viscosity scheme with respect to $W$ mappings for an infinite family of nonexpansive mappings

$$
x_{n+1}=\varepsilon_{n} r f\left(x_{n}\right)+\beta_{n} x_{n}+\left(\left(1-\beta_{n}\right) I-\varepsilon_{n} A\right) W_{n} J_{r}^{F} x_{n} .
$$

They prove that sequence $\left\{x_{n}\right\}$ and $J_{r_{n}}^{F}$ converge strongly to $z \in\left(\cap_{n=1}^{\infty} F\left(T_{n}\right)\right) \cap \operatorname{EP}(F)$, where $z$ is an equilibrium point for $F$ and is the unique solution of the variational inequality

$$
\langle(r f-A) z, x-z\rangle \leq 0, \quad \forall x \in \bigcap_{n=1}^{\infty} F\left(T_{n}\right) \cap \operatorname{EP}(F) .
$$

In 2012, Kangtunyakarn [35] modify the set of variational inequality to construct a new iterative scheme for finding a common element of the set of fixed point problems of infinite family of $k_{i}$ pseudocontractive mappings and the set of equilibrium problem and two sets of variational inequality problems. Let

$$
\begin{aligned}
F:= & \left(\bigcap_{i=1}^{\infty}\left\{F\left(T_{i}\right)\right\}\right) \cap\left(\bigcap_{k=1}^{M} \operatorname{SEP}\left(F_{k}\right)\right) \\
& \cap \operatorname{VI}(C, A) \cap \operatorname{VI}(C, B) .
\end{aligned}
$$

Starting with arbitrary initial point $x_{1} \in C$, define sequences $\left\{x_{n}\right\}$ and $\left\{u_{n}\right\}$ recursively by

$$
\begin{gathered}
F\left(u_{n}, y\right)+\frac{1}{r_{n}}\left\langle y-u_{n}, u_{n}-x_{n}\right\rangle \geq 0, \quad \forall y \in C, \\
x_{n+1}=\alpha_{n} u+\left(1-\alpha_{n}\right) S_{n} P_{C}\left(I-\gamma(a A+(1-a) B) u_{n}\right), \\
\forall n \in N,
\end{gathered}
$$

where $\left\{S_{n}: C \rightarrow C\right\}$ is the sequence defined by (37), $A, B$ is $\alpha$ and $\beta$-inverse-strongly monotone mapping, respectively, $a \in(0,1), 0<r<\min \{2 \alpha, 2 \beta\}$ and $\left\{r_{n}\right\} \subset[a, b] \subset$ $(0, \min \{2 \alpha, 2 \beta\})$. Under certain appropriate conditions they proved that the sequences $\left\{x_{n}\right\}$ and $\left\{u_{n}\right\}$ converge strongly to $z \in F$, where $z=P_{F} u$.

Let $A_{i}: C \rightarrow H$ be a mapping, for $i=1,2, \ldots, N$. By modification of (4), for $\delta_{i} \in(0,1)$, we have

$$
\begin{gathered}
\mathrm{VI}\left(C, \sum_{i=1}^{N} \delta_{i} A_{i}\right)=\left\{x^{*} \in C:\left\langle y-x^{*}, \sum_{i=1}^{N} \delta_{i} A_{i} x^{*}\right\rangle \geq 0,\right. \\
\left.\forall y \in C, \sum_{i=1}^{N} \delta_{i}=1\right\} .
\end{gathered}
$$

In this paper, motivated by the above results, we extend the nonexpansive mapping of hybrid steepest descent method (18) to nonexpansive semigroups and introduce a new iterative scheme for finding a common element of the set of solutions of a system of equilibrium problems, the set of fixed points of an infinite family of strictly pseudocontractive mappings, the set of solutions of fixed points for nonexpansive semigroups, and the set of solutions of variational inequality problems for relaxed cocoercive mapping in a real Hilbert space by the hybrid steepest descent method. The results shown in this paper improve and extend the recent ones announced by many others.

\section{Preliminaries}

Throughout this paper, we always assume that $C$ is a nonempty closed convex subset of a Hilbert space $H$. We write $x_{n} \rightarrow x$ to indicate that the sequence $\left\{x_{n}\right\}$ converges weakly to $x . x_{n} \rightarrow x$ implies that $\left\{x_{n}\right\}$ converges strongly to $x$. We denote by $N$ and $R$ the sets of positive integers and real numbers, respectively. For any $x \in H$, there exists a unique nearest point in $C$, denoted by $P_{C} x$, such that

$$
\left\|x-P_{C} x\right\| \leq\|x-y\|, \quad \forall y \in C .
$$


Such a $P_{C}$ is called the metric projection of $H$ onto $C$. It is known that $P_{C}$ is nonexpansive. Furthermore, for $x \in H$ and $u \in C$,

$$
u=P_{C} x \Longleftrightarrow\langle x-u, u-y\rangle \geq 0, \quad \forall y \in C .
$$

It is widely known that $H$ satisfies Opial's condition [8], that is, for any sequence $\left\{x_{n}\right\}$ with $x_{n} \rightarrow x$, the inequality

$$
\lim _{n \rightarrow \infty} \inf \left\|x_{n}-x\right\|<\lim _{n \rightarrow \infty} \inf \left\|x_{n}-y\right\|
$$

holds for every $y \in H$ with $y \neq x$.

In order to solve the equilibrium problem for a bifunction $F: C \times C \rightarrow R$, we assume that $F$ satisfies the following conditions:

(A1) $F(x, x)=0, \forall x \in C$,

(A2) $F$ is monotone, that is, $F(x, y)+F(y, x) \leq 0, \forall x, y \in$ C,

(A3) $\lim _{t \downarrow 0} F(t z+(1-t) x, y) \leq F(x, y), \forall x, y, z \in C$,

(A4) For each $x \in C, y \mapsto F(x, y)$ is convex and lower semicontinuous.

Let us recall the following lemmas which will be useful for our paper.

Lemma 3 (see [19]). Let $F$ be a bifunction from $C \times C$ into $R$ satisfying (A1), (A2), (A3), and (A4). Then, for any $r>0$ and $x \in H$, there exists $z \in C$ such that

$$
F(z, y)+\frac{1}{r}(y-z, z-x) \geq 0, \quad \forall y \in C .
$$

Furthermore, if $J_{r}^{F} x=\{z \in C: F(z, y)+(1 / r)(y-z, z-x) \geq$ $0, \forall y \in C\}$, then the following hold:

(1) $J_{r}^{F}$ is single-valued,

(2) $J_{r}^{F}$ is firmly nonexpansive, that is,

$$
\left\|J_{r}^{F} x-J_{r}^{F} y\right\|^{2} \leq\left\langle J_{r}^{F} x-J_{r}^{F} y, x-y\right\rangle, \quad \forall x, y \in H,
$$

(3) $F\left(J_{r}^{F}\right)=\operatorname{EP}(F)$,

(4) $\mathrm{EP}(F)$ is closed and convex.

Lemma 4 (see [12]). Let $C$ be a nonempty bounded closed and convex subset of a real Hilbert space $H$. Let $\Gamma=\left\{T(s): s \in R^{+}\right\}$ from $C$ be a nonexpansive semigroup on $C$, then for all $h>0$,

$$
\lim _{t \rightarrow \infty} \sup _{x \in C}\left\|\frac{1}{t} \int_{0}^{t} T(s) x d s-T(h)\left(\frac{1}{t} \int_{0}^{t} T(s) x d s\right)\right\|=0 .
$$

Lemma 5 (see [13]). Let $C$ be a nonempty bounded closed and convex subset of a real Hilbert space $H$, let $\left\{x_{n}\right\}$ be a sequence, and let $\Gamma=\left\{T(s): s \in R^{+}\right\}$from $C$ be a nonexpansive semigroup on $C$, if the following conditions are satisfied:

(i) $x_{n} \rightarrow z$,

(ii) $\lim \sup _{s \rightarrow \infty} \lim \sup _{n \rightarrow \infty}\left\|T(s) x_{n}-x_{n}\right\|=0$,

then, $z \in F(\Gamma)$.

Lemma 6 (see [36]). In a Hilbert space $H$, there holds the inequality

$$
\|x+y\|^{2} \leq\|x\|^{2}+2\langle y, x+y\rangle, \quad \forall x, y \in H .
$$

Lemma 7 (see [16]). Assume $A$ be a strongly positive linear bounded operator on $H$ with coefficient $\bar{\gamma}>0$ and $0 \leq \rho \geq$ $\|A\|^{-1}$, then $\|I-\rho A\| \leq 1-\rho \bar{\gamma}$.

Lemma 8 (see [37]). Let $B$ be a monotone mapping of $C$ into $H$ and let $N_{C} \omega_{1}$ be the normal cone to $C$ at $\omega_{1} \in C$, that is,

$$
N_{C} \omega_{1}=\left\{\omega \in H:\left\langle\omega_{1}-\omega_{2}, \omega\right\rangle \geq 0, \forall \omega_{2} \in C\right\},
$$

and define a mapping $Q$ on $C$ by

$$
Q \omega_{1}= \begin{cases}B \omega_{1}+N_{C} \omega_{1}, & \omega_{1} \in C, \\ \emptyset, & \omega_{1} \notin C .\end{cases}
$$

Then $Q$ is maximal monotone and $0 \in Q \omega_{1}$ if and only if, $\left\langle B \omega_{1}, \omega_{1}-\omega_{2}\right\rangle \geq 0$ for all $\omega_{2} \in C$.

Lemma 9 (see [27]). Let $\left\{x_{n}\right\}$ and $\left\{y_{n}\right\}$ be bounded sequences in a Banach space $E$ and $\left\{\gamma_{n}\right\}$ be a sequence in $[0,1]$ satisfying the following condition:

$$
0<\lim _{n \rightarrow \infty} \inf \gamma_{n} \leq \lim _{n \rightarrow \infty} \sup \gamma_{n}<1
$$

Suppose that $x_{n+1}=\gamma_{n} x_{n}+\left(1-\gamma_{n}\right) y_{n}, n \geq 0$ and $\lim _{n \rightarrow \infty} \sup \left(\left\|y_{n+1}-y_{n}\right\|-\left\|x_{n+1}-x_{n}\right\|\right) \leq 0$. Then $\lim _{n \rightarrow \infty} \| y_{n}-$ $x_{n} \|=0$.

Lemma 10 (see [28]). Assume that $\left\{a_{n}\right\}$ is a sequence of nonnegative real numbers such that

$$
a_{n+1} \leq\left(1-b_{n}\right) a_{n}+c_{n}, \quad n \geq 0
$$

where $\left\{b_{n}\right\}$ is a sequence in $(0,1)$ and $\left\{\delta_{n}\right\}$ is a sequence in $R$, such that

(i) $\sum_{i=1}^{\infty} b_{i}=\infty$,

(ii) $\lim _{n \rightarrow \infty} \sup \left(c_{n} / b_{n}\right) \leq 0$ or $\sum_{i=1}^{\infty}\left|c_{n}\right|<\infty$.

Then, $\lim _{n \rightarrow \infty} a_{n}=0$.

Let $C$ be a nonempty closed convex subset of a Hilbert space $H$. Let $\left\{T_{i}\right\}_{i=1}^{\infty}$ be mapping of $C$ into self. For all $j=$ $1,2, \ldots$, let $\rho_{j}=\left(\alpha_{1}^{j}, \alpha_{2}^{j}, \alpha_{2}^{j}\right) \in I \times I \times I$ where $I=[0,1]$ and 
$\alpha_{1}^{j}+\alpha_{2}^{j}+\alpha_{2}^{j}=1$. For every $n \in N$, we define the mapping $S_{n}: C \rightarrow C$ as follows:

$$
\begin{gathered}
U_{n, n+1}:=I, \\
U_{n, n}:=\alpha_{1}^{n} T_{n} U_{n, n+1}+\alpha_{2}^{n} U_{n, n+1}+\alpha_{3}^{n} I, \\
\vdots \\
U_{n, k}:=\alpha_{1}^{k} T_{k} U_{n, k+1}+\alpha_{2}^{k} U_{n, k+1}+\alpha_{3}^{k} I, \\
\vdots \\
U_{n, 2}:=\alpha_{1}^{2} T_{2} U_{n, 3}+\alpha_{2}^{2} U_{n, 3}+\alpha_{3}^{2} I, \\
S_{n}=U_{n, 1}:=\alpha_{1}^{1} T_{1} U_{n, 2}+\alpha_{2}^{1} U_{n, 2}+\alpha_{3}^{1} I .
\end{gathered}
$$

This mapping is called $S$-mapping generated by $T_{1}, \ldots, T_{n}$ and $\rho_{1}, \ldots, \rho_{n}$.

Lemma 11 (see [38]). Let $C$ be a nonempty closed convex subset of a Hilbert space $H$. Let $\left\{T_{i}\right\}_{i=1}^{\infty}$ be a $k_{i}$-strict pseudocontractive mapping of $C$ into self with $\kappa=\sup _{i} k_{i}$ and let $\rho_{j}=\left(\alpha_{1}^{j}, \alpha_{2}^{j}, \alpha_{2}^{j}\right) \in I \times I \times I$ where $I=[0,1], \alpha_{1}^{j}+\alpha_{2}^{j}+\alpha_{2}^{j}=1$, $\alpha_{1}^{j}+\alpha_{2}^{j} \leq b<1$, and $\alpha_{1}^{j}, \alpha_{2}^{j}, \alpha_{2}^{j} \in(\kappa, 1)$ for all $j=1,2, \ldots$. For every $n \in N$, let $S_{n}$ and $S$-mapping generated by $T_{1}, T_{2}, \ldots, T_{n}$ and $\rho_{1}, \rho_{2}, \ldots, \rho_{n}$ and $T_{1}, T_{2}, \ldots$, and $\rho_{1}, \rho_{2}, \ldots$, respectively. Then, for every $x \in C$ and $k \in N$, the limit $\lim _{n \rightarrow \infty} U_{n, k} x$ exists.

In view of the previous lemma, we will define the mapping $S: C \rightarrow C$ as follows:

$$
S x:=\lim _{n \rightarrow \infty} S_{n} x=\lim _{n \rightarrow \infty} U_{n, 1} x, \quad x \in C
$$

Remark 12 (see [38]). For each $n \in N, S_{n}$ is nonexpansive and $\lim _{n \rightarrow \infty} \sup _{x \in D}\left\|S_{n} x-S x\right\|=0$ for every bounded subset $D$ of C.

Lemma 13 (see [38]). Let $C$ be a nonempty closed convex subset of a Hilbert space $H$. Let $\left\{T_{i}\right\}_{i=1}^{\infty}$ be a $k_{i}$-strict pseudocontractive mapping of $C$ into self such that $\cap_{i=1}^{\infty} F\left(T_{i}\right) \neq \emptyset$ with $\kappa=\sup _{i} k_{i}$ and let $\rho_{j}=\left(\alpha_{1}^{j}, \alpha_{2}^{j}, \alpha_{2}^{j}\right) \in I \times I \times I$ where $I=[0,1], \alpha_{1}^{j}+\alpha_{2}^{j}+\alpha_{2}^{j}=1, \alpha_{1}^{j}+\alpha_{2}^{j} \leq b<1$, and $\alpha_{1}^{j}, \alpha_{2}^{j}, \alpha_{2}^{j} \in(\kappa, 1)$ for all $j=1,2, \ldots$. For every $n \in N$, let $S_{n}$ and $S$-mapping generated by $T_{1}, T_{2}, \ldots, T_{n}$ and $\rho_{1}, \rho_{2}, \ldots, \rho_{n}$, respectively. Then, $F(S)=\cap_{i=1}^{\infty} F\left(T_{i}\right) \neq \emptyset$.

\section{Main Results}

In this section, we will present our main results. To establish our results, we need the following technical lemmas.

Lemma 14. Let $C$ be a nonempty closed convex subset of a Hilbert space $H$ and let $A_{i}: C \rightarrow H$ be $\omega_{i}$-Lipschitz continuous and relaxed $\left(u_{i}, v_{i}\right)$-cocoercive mappings with $v_{i}-u_{i} \omega_{i}^{2}>0$, for $i=1,2, \ldots, N$. If $\cap_{i=1}^{N} \operatorname{VI}\left(C, A_{i}\right) \neq \emptyset$, then, for $\delta_{i} \in(0,1)$ and $\sum_{i=1}^{N} \delta_{i}=1$,

$$
\bigcap_{i=1}^{N} \mathrm{VI}\left(C, A_{i}\right)=\mathrm{VI}\left(C, \sum_{i=1}^{N} \delta_{i} A_{i}\right)
$$

Proof. The proof is by induction. This holds for $N=2$. In fact, for $a \in(0,1)$, it is obvious that

$$
\mathrm{VI}\left(C, A_{1}\right) \cap \mathrm{VI}\left(C, A_{2}\right) \subseteq \mathrm{VI}\left(C, \sum_{i=1}^{N} \delta_{i} A_{i}\right)
$$

Next, we will show that $\operatorname{VI}\left(C, \sum_{i=1}^{N} \delta_{i} A_{i}\right) \subseteq \operatorname{VI}\left(C, A_{1}\right) \cap$ $\mathrm{VI}\left(C, A_{2}\right)$.

Let

$$
\begin{gathered}
x_{0} \in \mathrm{VI}\left(C, \sum_{i=1}^{N} \delta_{i} A_{i}\right), \\
x^{*} \in \mathrm{VI}\left(C, A_{1}\right) \cap \mathrm{VI}\left(C, A_{2}\right) .
\end{gathered}
$$

It follows that

$$
\begin{aligned}
& \left\langle y-x^{*}, A_{1} x^{*}\right\rangle \geq 0, \quad \forall y \in C, \\
& \left\langle y-x^{*}, A_{2} x^{*}\right\rangle \geq 0, \quad \forall y \in C .
\end{aligned}
$$

Then, for every $a \in(0,1)$, one has

$$
\begin{gathered}
\left\langle y-x^{*}, a A_{1} x^{*}\right\rangle \geq 0, \quad \forall y \in C, \\
\left\langle y-x^{*},(1-a) A_{2} x^{*}\right\rangle \geq 0, \quad \forall y \in C .
\end{gathered}
$$

From $x_{0} \in \mathrm{VI}\left(C, \sum_{i=1}^{N} \delta_{i} A_{i}\right)$ and (43), one has

$$
\begin{aligned}
\left\langle x^{*}-x_{0}, a A_{1} x_{0}\right\rangle= & \left\langle x^{*}-x_{0},\left(\sum_{i=1}^{N} \delta_{i} A_{i}\right) x_{0}\right\rangle \\
& -\left\langle x^{*}-x_{0},(1-a) A_{2} x_{0}\right\rangle \\
\geq & (1-a)\left\langle x_{0}-x^{*}, A_{2} x_{0}\right\rangle \\
= & (1-a)\left\langle x_{0}-x^{*}, A_{2} x_{0}-A_{2} x^{*}\right\rangle \\
& +(1-a)\left\langle x_{0}-x^{*}, A_{2} x^{*}\right\rangle \\
\geq & (1-a)\left(v_{2}-u_{2} \omega_{2}^{2}\right)\left\|x_{0}-x^{*}\right\|^{2} \\
\geq & 0,
\end{aligned}
$$

which means

$$
\left\langle x^{*}-x_{0}, A_{1} x_{0}\right\rangle \geq 0 \text {. }
$$


On the other hand, from $x^{*} \in \mathrm{VI}\left(C, A_{1}\right)$, we have

$$
\begin{aligned}
\left\langle x^{*}-x_{0}, A_{1} x_{0}\right\rangle= & \left\langle x^{*}-x_{0}, A_{1} x_{0}-A_{1} x^{*}\right\rangle \\
& +\left\langle x^{*}-x_{0}, A_{1} x^{*}\right\rangle \\
\leq & \left\langle x^{*}-x_{0}, A_{1} x_{0}-A_{1} x^{*}\right\rangle \\
\leq & u_{1}\left\|A_{1} x_{0}-A_{1} x^{*}\right\|^{2}-v_{1}\left\|x_{0}-x^{*}\right\|^{2} \\
\leq & u_{1} \omega_{1}^{2}\left\|x_{0}-x^{*}\right\|^{2}-v_{1}\left\|x_{0}-x^{*}\right\|^{2} \\
= & \left(u_{1} \omega_{1}^{2}-v_{1}\right)\left\|x_{0}-x^{*}\right\|^{2} \\
\leq & 0 .
\end{aligned}
$$

This together with (46) leads to

$$
A_{1} x^{*}=A_{1} x_{0}
$$

Furthermore, for every $y \in C$, from (46) and (48), we obtain

$$
\begin{aligned}
\left\langle y-x_{0}, A_{1} x_{0}\right\rangle & =\left\langle y-x^{*}, A_{1} x_{0}\right\rangle+\left\langle x^{*}-x_{0}, A_{1} x_{0}\right\rangle \\
& \geq\left\langle y-x^{*}, A_{1} x_{0}\right\rangle \\
& =\left\langle y-x^{*}, A_{1} x^{*}\right\rangle \\
& \geq 0
\end{aligned}
$$

$$
x_{0} \in \mathrm{VI}\left(C, A_{1}\right) \text {. }
$$

It follows from (45) and (42) that

$$
\begin{aligned}
\left\langle x^{*}-x_{0},(1-a) A_{2} x_{0}\right\rangle \geq & \left\langle x_{0}-x^{*}, a A_{1} x_{0}\right\rangle \\
= & a\left\langle x_{0}-x^{*}, A_{1}\left(x_{0}-x^{*}\right)\right\rangle \\
& +a\left\langle x_{0}-x^{*}, A_{1} x^{*}\right\rangle \\
\geq & a\left\langle x_{0}-x^{*}, A_{1}\left(x_{0}-x^{*}\right)\right\rangle \\
\geq & a\left(v_{1}-u_{1} \omega_{1}^{2}\right)\left\|x_{0}-x^{*}\right\|^{2} \\
\geq & 0 .
\end{aligned}
$$

It yields that

$$
\left\langle x^{*}-x_{0}, A_{2} x_{0}\right\rangle \geq 0 \text {. }
$$

From $x^{*} \in \mathrm{VI}\left(C, A_{2}\right)$ and (52), one has

$$
\begin{aligned}
0 \leq & \left\langle x^{*}-x_{0}, A_{2} x_{0}\right\rangle \\
= & \left\langle x^{*}-x_{0}, A_{2} x_{0}-A_{2} x^{*}\right\rangle \\
& +\left\langle x^{*}-x_{0}, A_{2} x^{*}\right\rangle \\
\leq & \left\langle x^{*}-x_{0}, A_{2} x_{0}-A_{2} x^{*}\right\rangle \\
\leq & u_{2}\left\|A_{2} x_{0}-A_{2} x^{*}\right\|^{2}-v_{2}\left\|x_{0}-x^{*}\right\|^{2} \\
\leq & u_{2} \omega_{2}^{2}\left\|x_{0}-x^{*}\right\|^{2}-v_{1}\left\|x_{0}-x^{*}\right\|^{2} \\
= & \left(u_{2} \omega_{2}^{2}-v_{2}\right)\left\|x_{0}-x^{*}\right\|^{2} \\
\leq & 0 .
\end{aligned}
$$

That is,

$$
A_{2} x^{*}=A_{2} x_{0}
$$

Therefore, for every $y \in C$, from (52) and (54), we obtain

$$
\begin{aligned}
\left\langle y-x_{0}, A_{2} x_{0}\right\rangle & =\left\langle y-x^{*}, A_{2} x_{0}\right\rangle+\left\langle x^{*}-x_{0}, A_{2} x_{0}\right\rangle \\
& \geq\left\langle y-x^{*}, A_{2} x_{0}\right\rangle \\
& =\left\langle y-x^{*}, A_{2} x^{*}\right\rangle \\
& \geq 0,
\end{aligned}
$$

which means

$$
x_{0} \in \mathrm{VI}\left(C, A_{2}\right) \text {. }
$$

And hence,

$$
x_{0} \in \mathrm{VI}\left(C, A_{1}\right) \cap \mathrm{VI}\left(C, A_{2}\right) \text {. }
$$

Thus, we have

$$
\mathrm{VI}\left(C, \sum_{i=1}^{N} \delta_{i} A_{i}\right) \subseteq \mathrm{VI}\left(C, A_{1}\right) \cap \mathrm{VI}\left(C, A_{2}\right)
$$

Thus,

$$
\mathrm{VI}\left(C, \sum_{i=1}^{N} \delta_{i} A_{i}\right)=\mathrm{VI}\left(C, A_{1}\right) \cap \mathrm{VI}\left(C, A_{2}\right)
$$


Assume now that $\cap_{i=1}^{k} \mathrm{VI}\left(C, A_{i}\right)=\operatorname{VI}\left(C, \sum_{i=1}^{k} \delta_{i} A_{i}\right)$ is true for some $k$, and we show that it continues to hold for $k+1$. For $\delta_{i} \in(0,1)$ and $\sum_{i=1}^{k+1} \delta_{i}=1$, we have

$$
\begin{aligned}
& \mathrm{VI}\left(C, \sum_{i=1}^{k+1} \delta_{i} A_{i}\right) \\
& =\mathrm{VI}\left(C, \delta_{1} A_{1}+\sum_{i=2}^{k+1} \delta_{i} A_{i}\right) \\
& =\mathrm{VI}\left(C, \delta_{1} A_{1}+\left(1-\delta_{1}\right) \sum_{i=2}^{k+1} \frac{\delta_{i}}{1-\delta_{1}} A_{i}\right) \\
& =\mathrm{VI}\left(C, \delta_{1} A_{1}\right) \cap \mathrm{VI}\left(C,\left(1-\delta_{1}\right) \sum_{i=2}^{k+1} \frac{\delta_{i}}{1-\delta_{1}} A_{i}\right) \\
& =\mathrm{VI}\left(C, A_{1}\right) \cap \mathrm{VI}\left(C, \sum_{i=2}^{k+1} \frac{\delta_{i}}{1-\delta_{1}} A_{i}\right) \\
& =\operatorname{VI}\left(C, A_{1}\right) \cap\left(\bigcap_{i=2}^{k+1} \mathrm{VI}\left(C, A_{i}\right)\right) \\
& =\bigcap_{i=1}^{k+1} \mathrm{VI}\left(C, A_{i}\right) .
\end{aligned}
$$

By induction, $\cap_{i=1}^{k} \operatorname{VI}\left(C, A_{i}\right)=\operatorname{VI}\left(C, \sum_{i=1}^{k} \delta_{i} A_{i}\right)$ holds for $k=1,2, \ldots, N$ and this completes the proof.

Lemma 15. Let $C$ be a nonempty closed convex subset of a Hilbert space $H$, let $\Gamma=\left\{T(s): s \in R^{+}\right\}$from $C$ be a nonexpansive semigroup on $C$, and let $A_{i}: C \rightarrow H$ be $\omega_{i}$-Lipschitz continuous and relaxed $\left(\mu_{i}, v_{i}\right)$-cocoercive mappings with $v_{i}-$ $\mu_{i} \omega_{i}^{2}>0$, for $i=1,2, \ldots, N$. Assume that $D=\sum_{i=1}^{N} \delta_{i} A_{i}$, for $\delta_{i} \in(0,1)$ and $\sum_{i=1}^{N} \delta_{i}=1$. If $K_{n}(x)=\left(1 / t_{n}\right) \int_{0}^{t_{n}} T(s) S_{n} x d s$, where $\left\{S_{n}: C \rightarrow C\right\}$ is the sequence defined by (37) with $0 \leq \alpha_{n} \leq\left(2 \sum_{i=1}^{N} \delta_{i}\left(v_{i}-\mu_{i} \omega_{i}^{2}\right)\right) /\left(\sum_{i=1}^{N} \delta_{i} \omega_{i}^{2}\right)$, then $K_{n}-\alpha_{n} D K_{n}$ is a nonexpansive mapping in $H$. Furthermore, $I-\alpha_{n} D$ is a nonexpansive mapping in $H$.

Proof. Since $0 \leq \alpha_{n} \leq\left(2 \sum_{i=1}^{N} \delta_{i}\left(v_{i}-\mu_{i} \omega_{i}^{2}\right)\right) /\left(\sum_{i=1}^{N} \delta_{i} \omega_{i}^{2}\right)$, for every $x, y \in C$, we have

$$
\begin{aligned}
& \left\|\left(K_{n}-\alpha_{n} D K_{n}\right) x-\left(K_{n}-\alpha_{n} D K_{n}\right) y\right\|^{2} \\
& =\left\|\left(K_{n} x-K_{n} y\right)-\alpha_{n}\left(D K_{n} x-D K_{n} y\right)\right\|^{2} \\
& =\left\|K_{n} x-K_{n} y\right\|^{2}-2 \alpha_{n}\left\langle K_{n} x-K_{n} y, D K_{n} x-D K_{n} y\right\rangle \\
& \quad+\alpha_{n}^{2}\left\|D K_{n} x-D K_{n} y\right\|^{2} \\
& \leq\left\|K_{n} x-K_{n} y\right\|^{2}-2 \alpha_{n} \sum_{i=1}^{N} \delta_{i}\left(v_{i}-\mu_{i} \omega_{i}^{2}\right) \\
& \quad \times\left\|K_{n} x-K_{n} y\right\|^{2}+\alpha_{n}^{2} \sum_{i=1}^{N} \delta_{i} \omega_{i}^{2}\left\|K_{n} x-K_{n} y\right\|^{2}
\end{aligned}
$$

$$
\begin{aligned}
& =\left(1-2 \alpha_{n} \sum_{i=1}^{N} \delta_{i}\left(v_{i}-\mu_{i} \omega_{i}^{2}\right)+\alpha_{n}^{2} \sum_{i=1}^{N} \delta_{i} \omega_{i}^{2}\right) \\
& \quad \times\left\|K_{n} x-K_{n} y\right\|^{2} \\
& \leq\|x-y\|^{2} .
\end{aligned}
$$

Thus, we obtain that $K_{n}-\alpha_{n} D K_{n}$ is a nonexpansive mapping.

Similarly, we can obtain that $I-\alpha_{n} D$ is a nonexpansive mapping in $H$ and this completes the proof. 15.

The following main results follow from Lemmas 14 and

Theorem 16. Let $C$ be a nonempty closed convex subset of a real Hilbert space $H$, and let $F_{k}, k \in\{1,2, \ldots, M\}$ be a bifunction from $\mathrm{C} \times \mathrm{C} \rightarrow \mathrm{R}$ satisfying (A1)-(A4). Let $\Gamma=$ $\{T(s): 0 \leq s<\infty\}$ be a nonexpansive semigroup on $C$ and let $t_{n}$ be a positive real divergent sequence. Let $\left\{T_{i}\right\}_{i=1}^{\infty}$ be $k_{i}$-strict pseudo-contractive mappings of $C$ into self with $\kappa=\sup _{i} k_{i}$ and let $\rho_{j}=\left(\alpha_{1}^{j}, \alpha_{2}^{j}, \alpha_{2}^{j}\right) \in I \times I \times I$, where $I=[0,1]$, $\alpha_{1}^{j}+\alpha_{2}^{j}+\alpha_{2}^{j}=1, \alpha_{1}^{j}+\alpha_{2}^{j} \leq b<1$, and $\alpha_{1}^{j}, \alpha_{2}^{j}, \alpha_{2}^{j} \in(\kappa, 1)$ for all $j=1,2, \ldots$ For every $n \in N$, let $S_{n}$ and $S$-mapping generated by $T_{1}, T_{2}, \ldots, T_{n}$ and $\rho_{1}, \rho_{2}, \ldots, \rho_{n}$ with $T_{i}(F(\Gamma)) \subset F(\Gamma)$. Let $A_{i}: C \rightarrow H$ be $\omega_{i}$-Lipschitz continuous and relaxed $\left(\mu_{i}, v_{i}\right)$ cocoercive mappings with $v_{i}-\mu_{i} \omega_{i}^{2}>0$, for $i=1,2, \ldots, N$, let $f$ be a contraction of $H$ into itself with $\eta \in(0,1)$, and let $A$ be is a strongly positive linear bounded self-adjoint operator with the coefficients $\bar{\gamma}>0$ and $0<r<\bar{\gamma} / \eta$. Assume that

$$
\begin{aligned}
\Theta:= & F(\Gamma) \cap\left(\bigcap_{i=1}^{\infty}\left\{F\left(T_{i}\right)\right\}\right) \cap\left(\bigcap_{k=1}^{M} \operatorname{SEP}\left(F_{k}\right)\right) \\
& \cap\left(\bigcap_{i=1}^{N} \operatorname{VI}\left(C, A_{i}\right)\right) .
\end{aligned}
$$

Let $\left\{x_{n}\right\}$ be a sequence generated by $x_{1} \in C$ and

$$
\begin{gathered}
u_{n}=J_{r_{M, n}}^{F_{M}} J_{r_{M-1, n}}^{F_{M-1}} \cdots J_{r_{2, n}}^{F_{2}} J_{r_{1, n}}^{F_{1}} x_{n}, \\
z_{n}=P_{C}\left(\frac{1}{t_{n}} \int_{0}^{t_{n}} T(s) S_{n} u_{n} d s-\alpha_{n}\left(\sum_{i=1}^{N} \delta_{i} A_{i}\right)\right. \\
\left.\times \frac{1}{t_{n}} \int_{0}^{t_{n}} T(s) S_{n} u_{n} d s\right), \\
y_{n}=\varepsilon_{n} r f\left(x_{n}\right)+\beta_{n} x_{n}+\left(\left(1-\beta_{n}\right) I-\varepsilon_{n} A\right) z_{n}, \\
x_{n+1}=\gamma_{n} x_{n}+\left(1-\gamma_{n}\right) y_{n}, \quad \forall n \in N,
\end{gathered}
$$

where $\left\{S_{n}: C \rightarrow C\right\}$ is the sequence defined by (37) and $\delta_{i} \in$ $(0,1), \sum_{i=1}^{N} \delta_{i}=1$. If $\left\{\varepsilon_{n}\right\},\left\{\beta_{n}\right\}$ are two sequences in $(0,1)$ and 
$\left\{\gamma_{n}\right\} \subset\left[c_{1}, c_{2}\right] \subset(0,1)$ and $\left\{r_{k, n}\right\}$, for $k \in\{1,2, \ldots, M\}$ is a real sequence in $(0, \infty)$ satisfy the following conditions:

(i) $\lim _{n \rightarrow \infty} \varepsilon_{n}=0, \sum_{i=1}^{\infty} \varepsilon_{n}=\infty$,

(ii) $0<\lim _{n \rightarrow \infty} \inf \beta_{n} \leq \lim _{n \rightarrow \infty} \sup \beta_{n}<1$ and $\lim _{n \rightarrow \infty}\left|\beta_{n+1}-\beta_{n}\right|=0$,

(iii) $\lim _{n \rightarrow \infty} \inf r_{k, n}>0$ and $\lim _{n \rightarrow \infty}\left|r_{k, n+1}-r_{k, n}\right|=0$, for $k \in\{1,2, \ldots, M\}$,

(iv) $\left\{\alpha_{n}\right\} \subset\left[g_{1}, g_{2}\right] \subset\left(0,\left(2 \sum_{i=1}^{N} \delta_{i}\left(v_{i}-\mu_{i} \omega_{i}^{2}\right)\right) /\left(\sum_{i=1}^{N} \delta_{i} \omega_{i}^{2}\right)\right)$ and $\lim _{n \rightarrow \infty}\left|\alpha_{n+1}-\alpha_{n}\right|=0$,

(v) $\lim _{n \rightarrow \infty}\left|t_{n}-t_{n-1}\right| / t_{n}=0$.

Then $\left\{x_{n}\right\}$ converges strongly to $z \in \Theta$, where $z$ is the unique solution of variational inequality

$$
\lim _{n \rightarrow \infty} \sup \langle(r f-A) z, p-z\rangle \leq 0, \quad \forall p \in \Theta,
$$

which is the optimality condition for the minimization problem

$$
\min _{z \in \Theta} \frac{1}{2}\langle A z, z\rangle-h(z)
$$

where $h$ is a potential function for $r f\left(\right.$ i.e., $h^{\prime}(z)=r f(z)$ for $z \in H)$.

Proof. From the restrictions on control sequences, we may assume, without loss of generality, that $\varepsilon_{n} \leq\left(1-\beta_{n}\right)\|A\|^{-1}$ for all $n \geq 1$. From Lemma 7 , we know that if $0 \leq \rho \leq\|A\|^{-1}$, then $\|I-\rho A\| \leq 1-\rho \bar{\gamma}$. We will assume that $\|I-A\| \leq 1-\bar{\gamma}$. Since $A$ is a strongly positive linear bounded self-adjoint operator on $H$, we have

$$
\|A\|=\sup \{|\langle A x, x\rangle|: x \in H,\|x\|=1\} .
$$

Note that

$$
\begin{aligned}
\left\langle\left(\left(1-\beta_{n}\right) I-\varepsilon_{n} A\right) x, x\right\rangle & =1-\beta_{n}-\varepsilon_{n}\langle A x, x\rangle \\
& \geq 1-\beta_{n}-\varepsilon_{n}\|A\| \\
& \geq 0 .
\end{aligned}
$$

That is, $\left(1-\beta_{n}\right) I-\varepsilon_{n} A$ is positive. Furthermore,

$$
\begin{aligned}
& \left\|\left(1-\beta_{n}\right) I-\varepsilon_{n} A\right\| \\
& \quad=\sup \left\{\left|\left\langle\left(\left(1-\beta_{n}\right) I-\varepsilon_{n} A\right) x, x\right\rangle\right|: x \in H,\|x\|=1\right\} \\
& \quad=\sup \left\{1-\beta_{n}-\varepsilon_{n}\langle A x, x\rangle: x \in H,\|x\|=1\right\} \\
& \quad \leq 1-\beta_{n}-\varepsilon_{n} \bar{\gamma} .
\end{aligned}
$$

Next, We divide the proof of Theorem into five steps. Step 1. We show that $\left\{x_{n}\right\}$ is bounded.

Take $p \in \Theta$. Let $\Im_{n}^{k}=J_{r_{k, n}}^{F_{k}} J_{r_{k-1, n}}^{F_{k-1}} \cdots J_{r_{2, n}}^{F_{2}} J_{r_{1, n}}^{F_{1}}$, for $k \in$ $\{1,2, \ldots M\}$ and $\mathfrak{\Im}_{n}^{0}=I$, for any $n \in N$. Since $J_{r_{k, n}}^{F_{k}}$ is nonexpansive for each $k=1,2, \ldots M$ and $u_{n}=\Im_{n}^{M} x_{n}$, we have

$$
\left\|u_{n}-p\right\|=\left\|\Im_{n}^{M} x_{n}-\Im_{n}^{M} p\right\| \leq\left\|x_{n}-p\right\|
$$

From Lemma 15 and (69), one has

$$
\begin{gathered}
\left\|z_{n}-p\right\| \\
=\| P_{C}\left(\frac{1}{t_{n}} \int_{0}^{t_{n}} T(s) S_{n} u_{n} d s-\alpha_{n}\left(\sum_{i=1}^{N} \delta_{i} A_{i}\right)\right. \\
\left.\quad \times \frac{1}{t_{n}} \int_{0}^{t_{n}} T(s) S_{n} u_{n} d s\right)-p \| \\
=\| P_{C}\left(\frac{1}{t_{n}} \int_{0}^{t_{n}} T(s) S_{n} u_{n} d s-\alpha_{n}\left(\sum_{i=1}^{N} \delta_{i} A_{i}\right)\right. \\
\leq\left\|u_{n}-p\right\| \\
\left.\quad-\frac{1}{t_{n}} \int_{0}^{t_{n}} T(s) S_{n} u_{n} d s\right) \\
\quad-\left(\frac{1}{t_{n}} \int_{0}^{t_{n}} T(s) S_{n} p d s-\alpha_{n}\left(\sum_{i=1}^{N} \delta_{i} A_{i}\right)\right. \\
\quad \|\left(\frac{1}{t_{n}} \int_{0}^{t_{n}} T(s) S_{n} p d s-\alpha_{n}\left(\sum_{i=1}^{N} \delta_{i} A_{i}\right)\right. \\
\left.\quad \frac{1}{t_{n}} \int_{0}^{t_{n}} T(s) S_{n} S_{n} u_{n} d s\right) \\
\left.\quad \times \frac{1}{t_{n}} \int_{0}^{t_{n}} T(s) S_{n} p d s\right) \|
\end{gathered}
$$

It follows that

$$
\begin{aligned}
& \left\|y_{n}-p\right\| \\
& =\| \varepsilon_{n} \gamma f\left(x_{n}\right)+\beta_{n} x_{n} \\
& \quad+\left(\left(1-\beta_{n}\right) I-\varepsilon_{n} A\right) z_{n}-p \| \\
& =\| \varepsilon_{n}\left(\gamma f\left(x_{n}\right)-A p\right)+\beta_{n}\left(x_{n}-p\right) \\
& \quad+\left(\left(1-\beta_{n}\right) I-\varepsilon_{n} A\right)\left(z_{n}-p\right) \| \\
& \leq \varepsilon_{n}\left\|\gamma\left(f\left(x_{n}\right)-f(p)\right)+\gamma f(p)-A p\right\| \\
& +\beta_{n}\left\|x_{n}-p\right\|+\left(1-\beta_{n}-\varepsilon_{n} \bar{\gamma}\right)\left\|z_{n}-p\right\| \\
& \leq \varepsilon_{n} \gamma \eta\left\|x_{n}-p\right\|+\varepsilon_{n}\|\gamma f(p)-A p\| \\
& +\left(1-\varepsilon_{n} \bar{\gamma}\right)\left\|z_{n}-p\right\|
\end{aligned}
$$




$$
\begin{aligned}
\leq & \left(1-\varepsilon_{n}(\bar{\gamma}-\gamma \eta)\right)\left\|x_{n}-p\right\| \\
& +\varepsilon_{n}\|\gamma f(p)-A p\| .
\end{aligned}
$$

Furthermore,

$$
\begin{aligned}
\left\|x_{n+1}-p\right\|= & \left\|\gamma_{n} x_{n}+\left(1-\gamma_{n}\right) y_{n}-p\right\| \\
\leq & \gamma_{n}\left\|x_{n}-p\right\|+\left(1-\gamma_{n}\right)\left\|y_{n}-p\right\| \\
\leq & \left(1-\varepsilon_{n}\left(1-\gamma_{n}\right)(\bar{\gamma}-\gamma \eta)\right)\left\|x_{n}-p\right\| \\
& +\left(1-\gamma_{n}\right) \varepsilon_{n}\|\gamma f(p)-A p\| \\
\leq & \max \left\{\left\|x_{n}-p\right\|, \frac{\|\gamma f(p)-A p\|}{\bar{\gamma}-\gamma \eta}\right\} .
\end{aligned}
$$

By induction, we have

$$
\left\|x_{n}-p\right\| \leq \max \left\{\left\|x_{1}-p\right\|, \frac{\|\gamma f(p)-A p\|}{\bar{\gamma}-\gamma \eta}\right\}, \quad n \geq 1 .
$$

Hence, $\left\{x_{n}\right\}$ is bounded and we also obtain that $\left\{u_{n}\right\},\left\{z_{n}\right\}$, $\left\{y_{n}\right\},\left\{\left(\sum_{i=1}^{N} \delta_{i} A_{i}\right)\left(1 / t_{n}\right) \int_{0}^{t_{n}} T(s) S_{n} u_{n} d s\right\}$ and $\left\{f\left(x_{n}\right)\right\}$ are all bounded.

Step 2. We claim that $\lim _{n \rightarrow \infty}\left\|x_{n}-x_{n+1}\right\|=0$.

From the definition of $z_{n}$ and Lemma 15, for $p \in \Theta$, we have

$$
\begin{gathered}
\left\|z_{n+1}-z_{n}\right\| \\
=\| P_{C}\left(\frac{1}{t_{n+1}} \int_{0}^{t_{n+1}} T(s) S_{n+1} u_{n+1} d s-\alpha_{n+1}\left(\sum_{i=1}^{N} \delta_{i} A_{i}\right)\right. \\
\left.\quad \times \frac{1}{t_{n+1}} \int_{0}^{t_{n+1}} T(s) S_{n+1} u_{n+1} d s\right) \\
-P_{C}\left(\frac{1}{t_{n}} \int_{0}^{t_{n}} T(s) S_{n} u_{n} d s-\alpha_{n}\left(\sum_{i=1}^{N} \delta_{i} A_{i}\right)\right. \\
\leq \|\left(\frac{1}{t_{n+1}} \int_{0}^{t_{n+1}} T(s) S_{n+1} u_{n+1} d s-\alpha_{n+1}\left(\sum_{i=1}^{N} \delta_{i} A_{i}\right)\right. \\
\left.\quad \times \frac{1}{t_{n+1}} \int_{0}^{t_{n+1}} T(s) S_{n} u_{n} d s\right) \| \\
\quad-\left(\frac{1}{t_{n}} \int_{0}^{t_{n}} T(s) S_{n+1} u_{n+1} d s\right) \\
\left.\quad \times \frac{1}{t_{n}} \int_{0}^{t_{n}} T(s) S_{n} u_{n} d s\right) \|
\end{gathered}
$$

$$
\begin{aligned}
& \leq \|\left(I-\alpha_{n+1}\left(\sum_{i=1}^{N} \delta_{i} A_{i}\right)\right) \\
& \times\left(\frac{1}{t_{n+1}} \int_{0}^{t_{n+1}} T(s) S_{n+1} u_{n+1} d s-\frac{1}{t_{n}} \int_{0}^{t_{n}} T(s) S_{n} u_{n} d s\right) \| \\
& +\left\|\left(\alpha_{n}-\alpha_{n+1}\right)\left(\sum_{i=1}^{N} \delta_{i} A_{i}\right) \frac{1}{t_{n}} \int_{0}^{t_{n}} T(s) S_{n} u_{n} d s\right\| \\
& \leq\left\|\frac{1}{t_{n+1}} \int_{0}^{t_{n+1}} T(s) S_{n+1} u_{n+1} d s-\frac{1}{t_{n}} \int_{0}^{t_{n}} T(s) S_{n} u_{n} d s\right\| \\
& +\left|\alpha_{n}-\alpha_{n+1}\right|\left\|\left(\sum_{i=1}^{N} \delta_{i} A_{i}\right) \frac{1}{t_{n}} \int_{0}^{t_{n}} T(s) S_{n} u_{n} d s\right\| \\
& \leq\left\|\frac{1}{t_{n+1}} \int_{0}^{t_{n+1}} T(s)\left(S_{n+1} u_{n+1}-S_{n} u_{n}\right) d s\right\| \\
& +\|\left(\frac{1}{t_{n+1}}-\frac{1}{t_{n}}\right) \int_{0}^{t_{n}} T(s) S_{n} u_{n} d s \\
& +\frac{1}{t_{n+1}} \int_{t_{n}}^{t_{n+1}} T(s) S_{n} u_{n} d s \| \\
& +\left|\alpha_{n}-\alpha_{n+1}\right|\left\|\left(\sum_{i=1}^{N} \delta_{i} A_{i}\right) \frac{1}{t_{n}} \int_{0}^{t_{n}} T(s) S_{n} u_{n} d s\right\| \\
& \leq\left\|\frac{1}{t_{n+1}} \int_{0}^{t_{n+1}} T(s)\left(S_{n+1} u_{n+1}-S_{n} u_{n}\right) d s\right\| \\
& +\|\left(\frac{1}{t_{n+1}}-\frac{1}{t_{n}}\right) \int_{0}^{t_{n}} T(s)\left(S_{n} u_{n}-S_{n} p\right) d s \\
& +\frac{1}{t_{n+1}} \int_{t_{n}}^{t_{n+1}} T(s)\left(S_{n} u_{n}-S_{n} p\right) d s \| \\
& +\left|\alpha_{n}-\alpha_{n+1}\right|\left\|\left(\sum_{i=1}^{N} \delta_{i} A_{i}\right) \frac{1}{t_{n}} \int_{0}^{t_{n}} T(s) S_{n} u_{n} d s\right\| \\
& \leq\left\|S_{n+1} u_{n+1}-S_{n} u_{n}\right\|+\frac{2\left|t_{n+1}-t_{n}\right|}{t_{n+1}}\left\|u_{n}-p\right\| \\
& +\left|\alpha_{n}-\alpha_{n+1}\right|\left\|\left(\sum_{i=1}^{N} \delta_{i} A_{i}\right) \frac{1}{t_{n}} \int_{0}^{t_{n}} T(s) S_{n} u_{n} d s\right\| \\
& \leq\left\|u_{n+1}-u_{n}\right\|+\left\|S_{n+1} u_{n}-S_{n} u_{n}\right\| \\
& +\frac{2\left|t_{n+1}-t_{n}\right|}{t_{n+1}}\left\|u_{n}-p\right\| \\
& +\left|\alpha_{n}-\alpha_{n+1}\right|\left\|\left(\sum_{i=1}^{N} \delta_{i} A_{i}\right) \frac{1}{t_{n}} \int_{0}^{t_{n}} T(s) S_{n} u_{n} d s\right\| .
\end{aligned}
$$

First, we will show that if $\left\{x_{n}\right\}$ is bounded, then

$$
\lim _{n \rightarrow \infty}\left\|\Im_{n}^{k} x_{n}-\Im_{n+1}^{k} x_{n}\right\|=0
$$

for $k \in\{1,2, \ldots, M\}$. 
From Step 2 of the proof in [4], we have for $k \epsilon$ $\{1,2, \ldots, M\}$

$$
\lim _{n \rightarrow \infty}\left\|J_{r_{k, n+1}}^{F_{k}} x_{n}-J_{r_{k, n}}^{F_{k}} x_{n}\right\|=0
$$

For $k \in\{1,2, \ldots, M\}$, notice that

$$
\mathfrak{\Im}_{n}^{k}=J_{r_{k, n}}^{F_{k}} J_{r_{k-1, n}}^{F_{k-1}} \cdots J_{r_{2, n}}^{F_{2}} J_{r_{1, n}}^{F_{1}}=J_{r_{k, n}}^{F_{k}} \Im_{n}^{k-1} .
$$

It follows that

$$
\begin{aligned}
& \left\|\Im_{n}^{k} x_{n}-\Im_{n+1}^{k} x_{n}\right\| \\
& =\left\|J_{r_{k, n}}^{F_{k}} \mathfrak{\Im}_{n}^{k-1} x_{n}-J_{r_{k, n+1}}^{F_{k}} \mathfrak{\Im}_{n+1}^{k-1} x_{n}\right\| \\
& \leq\left\|J_{r_{k, n}}^{F_{k}} \mathfrak{\Im}_{n}^{k-1} x_{n}-J_{r_{k, n+1}}^{F_{k}} \mathfrak{\Im}_{n}^{k-1} x_{n}\right\| \\
& +\left\|J_{r_{k, n+1}}^{F_{k}} \Im_{n}^{k-1} x_{n}-J_{r_{k, n+1}}^{F_{k}} \Im_{n+1}^{k-1} x_{n}\right\| \\
& \leq\left\|J_{r_{k, n}}^{F_{k}} \Im_{n}^{k-1} x_{n}-J_{r_{k, n+1}}^{F_{k}} \Im_{n}^{k-1} x_{n}\right\| \\
& +\left\|J_{r_{k-1, n}}^{F_{k-1}} \mathfrak{\Im}_{n}^{k-2} x_{n}-J_{r_{k-1, n+1}}^{F_{k-1}} \mathfrak{\Im}_{n}^{k-2} x_{n}\right\| \\
& +\left\|\Im_{n}^{k-2} x_{n}-\Im_{n+1}^{k-2} x_{n}\right\| \\
& \leq\left\|J_{r_{k, n}}^{F_{k}} \Im_{n}^{k-1} x_{n}-J_{r_{k, n+1}}^{F_{k}} \Im_{n}^{k-1} x_{n}\right\| \\
& +\left\|J_{r_{k-1, n}}^{F_{k-1}} \Im_{n}^{k-2} x_{n}-J_{r_{k-1, n+1}}^{F_{k-1}} \Im_{n}^{k-2} x_{n}\right\| \\
& +\cdots+\left\|J_{r_{2, n}}^{F_{2}} \mathfrak{\Im}_{n}^{1} x_{n}-J_{r_{2, n+1}}^{F_{2}} \mathfrak{\Im}_{n}^{1} x_{n}\right\| \\
& +\left\|J_{r_{1, n}}^{F_{1}} x_{n}-J_{r_{1, n+1}}^{F_{1}} x_{n}\right\| .
\end{aligned}
$$

Therefore, from (76), we conclude (75).

Second, we estimate $\left\|u_{n+1}-u_{n}\right\|$. From $u_{n+1}=\mathfrak{\Im}_{n+1}^{M} x_{n+1}$ and $u_{n}=\mathfrak{\Im}_{n}^{M} x_{n}=J_{r_{M, n}}^{F_{M}} \mathfrak{\Im}_{n}^{M-1} x_{n}$, we obtain

$$
\begin{gathered}
F_{M}\left(u_{n+1}, y\right)+\frac{1}{r_{M, n+1}}\left\langle y-u_{n+1}, u_{n+1}-\Im_{n+1}^{M-1} x_{n+1}\right\rangle \\
\geq 0, \quad \forall y \in C \\
F_{M}\left(u_{n}, y\right)+\frac{1}{r_{M, n}}\left\langle y-u_{n}, u_{n}-\Im_{n}^{M-1} x_{n}\right\rangle \geq 0, \quad \forall y \in C .
\end{gathered}
$$

Taking $y=u_{n}$ in (79) and $y=u_{n+1}$ in (80), we have

$$
\begin{gathered}
F_{M}\left(u_{n+1}, u_{n}\right)+\frac{1}{r_{M, n+1}}\left\langle u_{n}-u_{n+1}, u_{n+1}-\Im_{n+1}^{M-1} x_{n+1}\right\rangle \geq 0, \\
F_{M}\left(u_{n}, u_{n+1}\right)+\frac{1}{r_{M, n}}\left\langle u_{n+1}-u_{n}, u_{n}-\Im_{n}^{M-1} x_{n}\right\rangle \geq 0 .
\end{gathered}
$$

So, from (A2), one has

$$
\left\langle u_{n+1}-u_{n}, \frac{u_{n}-\Im_{n}^{M-1} x_{n}}{r_{M, n}}-\frac{u_{n+1}-\Im_{n+1}^{M-1} x_{n+1}}{r_{M, n+1}}\right\rangle \geq 0 .
$$

Furthermore,

$$
\begin{aligned}
& \left\langle u_{n+1}-u_{n}, u_{n}-\Im_{n}^{M-1} x_{n}-\left(u_{n+1}-\Im_{n+1}^{M-1} x_{n+1}\right)\right. \\
& \left.+\left(1-\frac{r_{M, n}}{r_{M, n+1}}\right)\left(u_{n+1}-\Im_{n+1}^{M-1} x_{n+1}\right)\right\rangle \geq 0 .
\end{aligned}
$$

Since $\lim _{n \rightarrow \infty} r_{k, n}>0$, we assume that there exists a real number such that $r_{k, n}>a>0$ for all $n \in N$. Thus, we obtain

$$
\begin{aligned}
& \left\|u_{n+1}-u_{n}\right\| \\
& \leq\left\|\Im_{n}^{M-1} x_{n}-\Im_{n+1}^{M-1} x_{n+1}\right\| \\
& +\left|1-\frac{r_{M, n}}{r_{M, n+1}}\right|\left\|u_{n+1}-\Im_{n+1}^{M-1} x_{n+1}\right\| \\
& \leq\left\|\mathfrak{\Im}_{n}^{M-1} x_{n}-\mathfrak{\Im}_{n+1}^{M-1} x_{n}\right\|+\left\|\Im_{n+1}^{M-1} x_{n}-\mathfrak{\Im}_{n+1}^{M-1} x_{n+1}\right\| \\
& +\frac{1}{a}\left|r_{M, n+1}-r_{M, n}\right|\left\|u_{n+1}-\Im_{n+1}^{M-1} x_{n+1}\right\| \\
& \leq\left\|\mathfrak{J}_{n}^{M-1} x_{n}-\mathfrak{\Im}_{n+1}^{M-1} x_{n}\right\|+\left\|x_{n}-x_{n+1}\right\| \\
& +\frac{1}{a}\left|r_{M, n+1}-r_{M, n}\right|\left\|u_{n+1}-\Im_{n+1}^{M-1} x_{n+1}\right\| .
\end{aligned}
$$

Third, we estimate $\left\|S_{n+1} u_{n}-S_{n} u_{n}\right\|$. It follows from (37) that

$$
\begin{aligned}
& \left\|S_{n+1} u_{n}-S_{n} u_{n}\right\|^{2} \\
& =\left\|U_{n+1,1} u_{n}-U_{n, 1} u_{n}\right\|^{2} \\
& =\| \alpha_{1}^{1} T_{1} U_{n+1,2} u_{n}+\alpha_{2}^{1} U_{n+1,2} u_{n}+\alpha_{3}^{1} u_{n} \\
& -\left(\alpha_{1}^{1} T_{1} U_{n, 2} u_{n}+\alpha_{2}^{1} U_{n, 2} u_{n}+\alpha_{3}^{1} u_{n}\right) \|^{2} \\
& =\| \alpha_{1}^{1}\left(T_{1} U_{n+1,2}-T_{1} U_{n, 2}\right) u_{n} \\
& +\alpha_{2}^{1}\left(U_{n+1,2}-U_{n, 2}\right) u_{n} \|^{2} \\
& \leq \alpha_{1}^{1}\left\|\left(T_{1} U_{n+1,2}-T_{1} U_{n, 2}\right) u_{n}\right\|^{2} \\
& +\alpha_{2}^{1}\left\|\left(U_{n+1,2}-U_{n, 2}\right) u_{n}\right\|^{2} \\
& -\alpha_{1}^{1} \alpha_{2}^{1} \|\left(T_{1} U_{n+1,2}-T_{1} U_{n, 2}\right) u_{n} \\
& -\left(U_{n+1,2}-U_{n, 2}\right) u_{n} \|^{2} \\
& \leq \alpha_{1}^{1}\left(\left\|U_{n+1,2} u_{n}-U_{n, 2} u_{n}\right\|^{2}\right. \\
& \left.+\kappa\left\|\left(I-T_{1}\right) U_{n+1,2} u_{n}-\left(I-T_{1}\right) U_{n, 2} u_{n}\right\|^{2}\right) \\
& +\alpha_{1}^{2}\left\|U_{n+1,2} u_{n}-U_{n, 2} u_{n}\right\|^{2} \\
& -\alpha_{1}^{1} \alpha_{2}^{1}\left\|\left(I-T_{1}\right) U_{n+1,2} u_{n}-\left(I-T_{1}\right) U_{n, 2} u_{n}\right\|^{2}
\end{aligned}
$$




$$
\begin{aligned}
& \leq\left(1-\alpha_{1}^{3}\right)\left\|U_{n+1,2} u_{n}-U_{n, 2} u_{n}\right\|^{2} \\
& \vdots \\
& \leq \prod_{i=1}^{n}\left(1-\alpha_{3}^{i}\right)\left\|U_{n+1, n+1} u_{n}-U_{n, n+1} u_{n}\right\|^{2},
\end{aligned}
$$

which means that

$$
\left\|S_{n+1} u_{n}-S_{n} u_{n}\right\| \leq L_{1} \prod_{i=1}^{n}\left(1-\alpha_{3}^{i}\right),
$$

where $L_{1} \geq 0$ is a constant such that $\left\|U_{n+1, n+1} u_{n}-U_{n, n+1} u_{n}\right\| \leq$ $L_{1}$, for all $n \in N$.

Next, we estimate $\left\|y_{n+1}-y_{n}\right\|$. Substituting (84) and (86) into (74), one has

$$
\begin{aligned}
& \left\|z_{n+1}-z_{n}\right\| \\
& \leq\left\|x_{n}-x_{n+1}\right\|+\left\|\mathfrak{J}_{n}^{M-1} x_{n}-\Im_{n+1}^{M-1} x_{n}\right\| \\
& \quad+\frac{1}{a}\left|r_{M, n+1}-r_{M, n}\right|\left\|u_{n+1}-\Im_{n+1}^{M-1} x_{n+1}\right\| \\
& \quad+L_{1} \prod_{i=1}^{n}\left(1-\alpha_{3}^{i}\right) \\
& \quad+\left|\alpha_{n}-\alpha_{n+1}\right|\left\|\left(\sum_{i=1}^{N} \delta_{i} A_{i}\right) \frac{1}{t_{n}} \int_{0}^{t_{n}} T(s) S_{n} u_{n} d s\right\| \\
& \quad+\frac{2\left|t_{n+1}-t_{n}\right|}{t_{n+1}}\left\|u_{n}-p\right\| .
\end{aligned}
$$

From (61), we have

$$
\begin{aligned}
& \left\|y_{n+1}-y_{n}\right\| \\
& =\| \varepsilon_{n+1} \gamma\left(f\left(x_{n+1}\right)-f\left(x_{n}\right)\right) \\
& \quad+\left(\varepsilon_{n+1}-\varepsilon_{n}\right)\left(\gamma f\left(x_{n}\right)-A z_{n}\right) \\
& \quad+\beta_{n+1}\left(x_{n+1}-x_{n}\right)+\left(\beta_{n+1}-\beta_{n}\right)\left(x_{n}-z_{n}\right) \\
& \quad+\left(\left(1-\beta_{n+1}\right) I-\varepsilon_{n+1} A\right)\left(z_{n+1}-z_{n}\right) \| \\
& \leq \varepsilon_{n+1} \gamma \eta\left\|x_{n+1}-x_{n}\right\|+\left|\varepsilon_{n+1}-\varepsilon_{n}\right| \\
& \quad \times \quad\left\|\gamma f\left(x_{n}\right)-A z_{n}\right\| \\
& +\beta_{n+1}\left\|x_{n+1}-x_{n}\right\|+\left|\beta_{n+1}-\beta_{n}\right|\left\|x_{n}-z_{n}\right\| \\
& +\left(1-\beta_{n+1}-\varepsilon_{n+1} \bar{\gamma}\right)\left\|z_{n+1}-z_{n}\right\| .
\end{aligned}
$$

Substitution (87) into (88) yields that

$$
\begin{aligned}
& \left\|y_{n+1}-y_{n}\right\| \\
& \leq\left(1-\varepsilon_{n+1}(\bar{\gamma}-\gamma \eta)\right)\left\|x_{n+1}-x_{n}\right\| \\
& \quad+L_{2}\left(\left|\varepsilon_{n+1}-\varepsilon_{n}\right|+\left|\beta_{n+1}-\beta_{n}\right|+\frac{2\left|t_{n+1}-t_{n}\right|}{t_{n+1}}\right. \\
& \left.\quad+\left|r_{M, n+1}-r_{M, n}\right|+\left|\alpha_{n}-\alpha_{n+1}\right|\right) \\
& +\left\|\mathfrak{\Im}_{n}^{M-1} x_{n}-\Im_{n+1}^{M-1} x_{n}\right\|+L_{1} \prod_{i=1}^{n}\left(1-\alpha_{3}^{i}\right),
\end{aligned}
$$

where $L_{2}$ is an appropriate constant such that

$$
\begin{gathered}
L_{2}=\max \left\{\sup _{n \geq 1}\left\{\left\|\left(\sum_{i=1}^{N} \delta_{i} A_{i}\right) \frac{1}{t_{n}} \int_{0}^{t_{n}} T(s) S_{n} u_{n} d s\right\|\right\},\right. \\
\sup _{n \geq 1}\left\{\frac{1}{a}\left\|u_{n+1}-\Im_{n+1}^{M-1} x_{n+1}\right\|\right\}, \\
\sup _{n \geq 1}\left\{\left\|\gamma f\left(x_{n}\right)-A z_{n}\right\|\right\}, \sup _{n \geq 1}\left\{\left\|x_{n}-z_{n}\right\|\right\}, \\
\left.\sup _{n \geq 1}\left\{\left\|u_{n}-p\right\|\right\}\right\} .
\end{gathered}
$$

It follows from (89) that

$$
\begin{aligned}
& \left\|y_{n+1}-y_{n}\right\|-\left\|x_{n+1}-x_{n}\right\| \\
& \leq L_{2}\left(\left|\varepsilon_{n+1}-\varepsilon_{n}\right|+\left|\beta_{n+1}-\beta_{n}\right|+\frac{2\left|t_{n+1}-t_{n}\right|}{t_{n+1}}\right. \\
& \left.+\left|r_{M, n+1}-r_{M, n}\right|+\left|\alpha_{n}-\alpha_{n+1}\right|\right) \\
& +\left\|\mathfrak{\Im}_{n}^{M-1} x_{n}-\Im_{n+1}^{M-1} x_{n}\right\|+L_{1} \prod_{i=1}^{n}\left(1-\alpha_{3}^{i}\right) .
\end{aligned}
$$

Consequently, from (75) and the conditions in Theorem 16, we obtain

$$
\lim _{n \rightarrow \infty} \sup \left(\left\|y_{n+1}-y_{n}\right\|-\left\|x_{n+1}-x_{n}\right\|\right) \leq 0 .
$$

Hence, by Lemma 9, one has

$$
\lim _{n \rightarrow \infty}\left\|y_{n}-x_{n}\right\|=0 \text {. }
$$

Since $x_{n+1}=\gamma_{n} x_{n}+\left(1-\gamma_{n}\right) y_{n}$, this shows that

$$
\lim _{n \rightarrow \infty}\left\|x_{n+1}-x_{n}\right\|=\lim _{n \rightarrow \infty}\left(1-\gamma_{n}\right)\left\|y_{n}-x_{n}\right\|=0 \text {. }
$$

Step 3. We claim that $\lim _{n \rightarrow \infty}\left\|\left(1 / t_{n}\right) \int_{0}^{t_{n}} T(s) S_{n} u_{n} d s-u_{n}\right\|=0$. Observing $y_{n}=\varepsilon_{n} \gamma f\left(x_{n}\right)+\beta_{n} x_{n}+\left(\left(1-\beta_{n}\right) I-\varepsilon_{n} A\right) z_{n}$, we obtain

$$
\begin{aligned}
\left\|x_{n}-z_{n}\right\| \leq & \left\|x_{n}-y_{n}\right\|+\left\|y_{n}-z_{n}\right\| \\
\leq & \left\|x_{n}-y_{n}\right\|+\varepsilon_{n}\left\|\gamma f\left(x_{n}\right)-A z_{n}\right\| \\
& +\beta_{n}\left\|x_{n}-z_{n}\right\|,
\end{aligned}
$$


12

Abstract and Applied Analysis

which means that

$$
\left\|x_{n}-z_{n}\right\| \leq \frac{1}{1-\beta_{n}}\left\|x_{n}-y_{n}\right\|+\frac{\varepsilon_{n}}{1-\beta_{n}}\left\|\gamma f\left(x_{n}\right)-A z_{n}\right\| .
$$

This together with the conditions (i) and (ii) imply that

$$
\lim _{n \rightarrow \infty}\left\|x_{n}-z_{n}\right\|=0
$$

From (93) and (97), one has

$$
\lim _{n \rightarrow \infty}\left\|y_{n}-z_{n}\right\| \leq \lim _{n \rightarrow \infty}\left(\left\|y_{n}-x_{n}\right\|+\left\|x_{n}-z_{n}\right\|\right)=0
$$

For $p \in \Theta$, we see that

$$
\left\|z_{n}-p\right\|^{2} \quad \times\left\|A_{i}\left(\frac{1}{t_{n}} \int_{0}^{t_{n}} T(s) S_{n} u_{n} d s-\frac{1}{t_{n}} \int_{0}^{t_{n}} T(s) S_{n} p d s\right)\right\|^{2} .
$$$$
=\| P_{C}\left(\frac{1}{t_{n}} \int_{0}^{t_{n}} T(s) S_{n} u_{n} d s-\alpha_{n}\left(\sum_{i=1}^{N} \delta_{i} A_{i}\right)\right.
$$$$
\left.\times \frac{1}{t_{n}} \int_{0}^{t_{n}} T(s) S_{n} u_{n} d s\right)-p \|^{2}
$$$$
=\| P_{C}\left(\frac{1}{t_{n}} \int_{0}^{t_{n}} T(s) S_{n} u_{n} d s-\alpha_{n}\left(\sum_{i=1}^{N} \delta_{i} A_{i}\right)\right.
$$$$
\left.\times \frac{1}{t_{n}} \int_{0}^{t_{n}} T(s) S_{n} u_{n} d s\right)
$$$$
-P_{C}\left(\frac{1}{t_{n}} \int_{0}^{t_{n}} T(s) S_{n} p d s-\alpha_{n}\left(\sum_{i=1}^{N} \delta_{i} A_{i}\right)\right.
$$$$
\left.\times \frac{1}{t_{n}} \int_{0}^{t_{n}} T(s) S_{n} p d s\right) \|^{2}
$$$$
\leq \|\left(\frac{1}{t_{n}} \int_{0}^{t_{n}} T(s) S_{n} u_{n} d s-\frac{1}{t_{n}} \int_{0}^{t_{n}} T(s) S_{n} p d s\right)
$$$$
-\alpha_{n}\left(\sum_{i=1}^{N} \delta_{i} A_{i}\right)
$$$$
\times\left(\frac{1}{t_{n}} \int_{0}^{t_{n}} T(s) S_{n} u_{n} d s-\frac{1}{t_{n}} \int_{0}^{t_{n}} T(s) S_{n} p d s\right) \|^{2}
$$$$
=\left\|\left(\frac{1}{t_{n}} \int_{0}^{t_{n}} T(s) S_{n} u_{n} d s-\frac{1}{t_{n}} \int_{0}^{t_{n}} T(s) S_{n} p d s\right)\right\|^{2}
$$$$
-2 \alpha_{n}\left\langle\left(\frac{1}{t_{n}} \int_{0}^{t_{n}} T(s) S_{n} u_{n} d s\right.\right.
$$$$
\left.-\frac{1}{t_{n}} \int_{0}^{t_{n}} T(s) S_{n} p d s\right)
$$

$$
\begin{aligned}
\left(\sum_{i=1}^{N} \delta_{i} A_{i}\right) & \frac{1}{t_{n}} \int_{0}^{t_{n}} T(s) S_{n} u_{n} d s \\
& \left.\left.-\frac{1}{t_{n}} \int_{0}^{t_{n}} T(s) S_{n} p d s\right)\right\rangle
\end{aligned}
$$

$$
+\alpha_{n}^{2} \|\left(\sum_{i=1}^{N} \delta_{i} A_{i}\right)
$$

$$
\begin{gathered}
\times\left(\left(\frac{1}{t_{n}} \int_{0}^{t_{n}} T(s) S_{n} u_{n} d s\right.\right. \\
\left.\left.\quad-\frac{1}{t_{n}} \int_{0}^{t_{n}} T(s) S_{n} p d s\right)\right) \|^{2}
\end{gathered}
$$$$
\leq\left\|x_{n}-p\right\|^{2}-\alpha_{n} \sum_{i=1}^{N} \delta_{i}\left(\frac{2 v_{i}}{\omega_{i}^{2}}-2 \mu_{i}-\alpha_{n}\right)
$$

It follows from (42) that

$$
\begin{aligned}
\| y_{n}- & p \|^{2} \\
= & \| \varepsilon_{n} r f\left(x_{n}\right)+\beta_{n} x_{n} \\
& +\left(\left(1-\beta_{n}\right) I-\varepsilon_{n} A\right) z_{n}-p \|^{2} \\
= & \|\left(\left(1-\beta_{n}\right) I-\varepsilon_{n} A\right)\left(z_{n}-p\right) \\
& +\beta_{n}\left(x_{n}-p\right)+\varepsilon_{n}\left(r f\left(x_{n}\right)-A p\right) \|^{2} \\
= & \left(\left(1-\beta_{n}\right) I-\varepsilon_{n} A\right)\left(z_{n}-p\right)+\beta_{n}\left(x_{n}-p\right) \|^{2} \\
& +\varepsilon_{n}^{2}\left\|r f\left(x_{n}\right)-A p\right\|^{2} \\
& +2 \varepsilon_{n}\left\langle\left(\left(1-\beta_{n}\right) I-\varepsilon_{n} A\right)\left(z_{n}-p\right),\right. \\
& \left.\quad r f\left(x_{n}\right)-A p\right\rangle \\
& +2 \beta_{n} \varepsilon_{n}\left\langle x_{n}-p, r f\left(x_{n}\right)-A p\right\rangle \\
\leq & \left(\left(1-\beta_{n}-\varepsilon_{n} \bar{\gamma}\right)\left\|z_{n}-p\right\|+\beta_{n}\left\|x_{n}-p\right\|\right)^{2} \\
& +\varepsilon_{n}^{2}\left\|r f\left(x_{n}\right)-A p\right\|^{2} \\
& +2 \varepsilon_{n}\left\langle\left(\left(1-\beta_{n}\right) I-\varepsilon_{n} A\right)\left(z_{n}-p\right),\right. \\
& +\beta_{n}^{2}\left\|x_{n}-p\right\|^{2}+2 \beta_{n}\left(1-\beta_{n}-\varepsilon_{n} \bar{\gamma}\right) \\
& +2 \beta_{n} \varepsilon_{n}\left\langle x_{n}-p, r f\left(x_{n}\right)-A p\right\rangle \\
& \left(1-\beta_{n}-\varepsilon_{n} \bar{\gamma}\right)^{2}\left\|z_{n}-p\right\|^{2} \\
&
\end{aligned}
$$




$$
\begin{aligned}
& \times\left\|z_{n}-p\right\|\left\|x_{n}-p\right\| \\
+ & \varepsilon_{n}^{2}\left\|r f\left(x_{n}\right)-A p\right\|^{2}+2 \varepsilon_{n} \\
\times & \left\langle\left(\left(1-\beta_{n}\right) I-\varepsilon_{n} A\right)\left(z_{n}-p\right),\right. \\
& \left.r f\left(x_{n}\right)-A p\right\rangle \\
+ & 2 \beta_{n} \varepsilon_{n}\left\langle x_{n}-p, r f\left(x_{n}\right)-A p\right\rangle \\
\leq & \left(1-\beta_{n}-\varepsilon_{n} \bar{\gamma}\right)^{2}\left\|z_{n}-p\right\|^{2}+\beta_{n}^{2}\left\|x_{n}-p\right\|^{2} \\
+ & \beta_{n}\left(1-\beta_{n}-\varepsilon_{n} \bar{\gamma}\right) \\
\times & \left(\left\|z_{n}-p\right\|^{2}+\left\|x_{n}-p\right\|^{2}\right) \\
+ & \varepsilon_{n}^{2}\left\|r f\left(x_{n}\right)-A p\right\|^{2}+2 \varepsilon_{n} \\
\times & \left\langle\left(\left(1-\beta_{n}\right) I-\varepsilon_{n} A\right)\left(z_{n}-p\right),\right. \\
& \left.r f\left(x_{n}\right)-A p\right\rangle \\
+ & 2 \beta_{n} \varepsilon_{n}\left\langle x_{n}-p, r f\left(x_{n}\right)-A p\right\rangle \\
= & \left(1-\varepsilon_{n} \bar{\gamma}\right)\left(1-\beta_{n}-\varepsilon_{n} \bar{\gamma}\right)\left\|z_{n}-p\right\|^{2} \\
+ & \left(1-\varepsilon_{n} \bar{\gamma}\right) \beta_{n}\left\|x_{n}-p\right\|^{2} \\
+ & \varepsilon_{n}^{2}\left\|r f\left(x_{n}\right)-A p\right\|^{2}+2 \varepsilon_{n} \\
\times & \left\langle\left(\left(1-\beta_{n}\right) I-\varepsilon_{n} A\right)\left(z_{n}-p\right),\right. \\
& \left.\quad r f\left(x_{n}\right)-A p\right\rangle \\
+ & 2 \beta_{n}\left\langle x_{n}-p, r f\left(x_{n}\right)-A p\right\rangle . \\
&
\end{aligned}
$$

Substituting (99) into (100) yields that

$$
\begin{aligned}
& \left\|y_{n}-p\right\|^{2} \\
& \leq\left(1-\varepsilon_{n} \bar{\gamma}\right)\left(1-\beta_{n}-\varepsilon_{n} \bar{\gamma}\right) \\
& \times\left\{\left\|x_{n}-p\right\|^{2}-a \alpha_{n}\left(\frac{2 v_{1}}{\omega_{1}^{2}}-2 \mu_{1}+\alpha_{n}\right)\right. \\
& \times \| A_{1}\left(\frac{1}{t_{n}} \int_{0}^{t_{n}} T(s) S_{n} u_{n} d s\right. \\
& \left.-\frac{1}{t_{n}} \int_{0}^{t_{n}} T(s) S_{n} p d s\right) \|^{2} \\
& -(1-a) \alpha_{n}\left(\frac{2 v_{2}}{\omega_{2}^{2}}-2 \mu_{2}+\alpha_{n}\right) \\
& \times \| A_{2}\left(\frac{1}{t_{n}} \int_{0}^{t_{n}} T(s) S_{n} u_{n} d s\right. \\
& \left.\left.-\frac{1}{t_{n}} \int_{0}^{t_{n}} T(s) S_{n} p d s\right) \|^{2}\right\}
\end{aligned}
$$

$$
\begin{aligned}
& +\left(1-\varepsilon_{n} \bar{\gamma}\right) \beta_{n}\left\|x_{n}-p\right\|^{2} \\
& +\varepsilon_{n}^{2}\left\|r f\left(x_{n}\right)-A p\right\|^{2} \\
& +2 \varepsilon_{n}\left\langle\left(\left(1-\beta_{n}\right) I-\varepsilon_{n} A\right)\left(z_{n}-p\right),\right. \\
& \left.r f\left(x_{n}\right)-A p\right\rangle \\
& +2 \beta_{n} \varepsilon_{n}\left\langle x_{n}-p, r f\left(x_{n}\right)-A p\right\rangle \\
& =\left(1-\varepsilon_{n} \bar{\gamma}\right)^{2}\left\|x_{n}-p\right\|^{2} \\
& +\left(1-\varepsilon_{n} \bar{\gamma}\right)\left(1-\beta_{n}-\varepsilon_{n} \bar{\gamma}\right) \\
& \times\left\{-\alpha_{n} \sum_{i=1}^{N} \delta_{i}\left(\frac{2 v_{i}}{\omega_{i}^{2}}-2 \mu_{i}-\alpha_{n}\right)\right. \\
& \times \| A_{i}\left(\frac{1}{t_{n}} \int_{0}^{t_{n}} T(s) S_{n} u_{n} d s\right. \\
& \left.\left.-\frac{1}{t_{n}} \int_{0}^{t_{n}} T(s) S_{n} p d s\right) \|^{2}\right\} \\
& +2 \varepsilon_{n}\left\langle\left(\left(1-\beta_{n}\right) I-\varepsilon_{n} A\right)\left(z_{n}-p\right),\right. \\
& \left.r f\left(x_{n}\right)-A p\right\rangle \\
& +2 \beta_{n} \varepsilon_{n}\left\langle x_{n}-p, r f\left(x_{n}\right)-A p\right\rangle \\
& +\varepsilon_{n}^{2}\left\|r f\left(x_{n}\right)-A p\right\|^{2} \\
& \leq\left\|x_{n}-p\right\|^{2}+\left(1-\varepsilon_{n} \bar{\gamma}\right)\left(1-\beta_{n}-\varepsilon_{n} \bar{\gamma}\right) \\
& \times\left\{-\alpha_{n} \sum_{i=1}^{N} \delta_{i}\left(\frac{2 v_{i}}{\omega_{i}^{2}}-2 \mu_{i}-\alpha_{n}\right)\right.
\end{aligned}
$$$$
\times \| A_{i}\left(\frac{1}{t_{n}} \int_{0}^{t_{n}} T(s) S_{n} u_{n} d s\right.
$$$$
\left.\left.-\frac{1}{t_{n}} \int_{0}^{t_{n}} T(s) S_{n} p d s\right) \|^{2}\right\}
$$$$
+2 \varepsilon_{n}\left\langle\left(\left(1-\beta_{n}\right) I-\varepsilon_{n} A\right)\left(z_{n}-p\right),\right.
$$$$
\left.r f\left(x_{n}\right)-A p\right\rangle
$$$$
+2 \beta_{n} \varepsilon_{n}\left\langle x_{n}-p, r f\left(x_{n}\right)-A p\right\rangle
$$$$
+\varepsilon_{n}^{2}\left\|r f\left(x_{n}\right)-A p\right\|^{2} \text {. }
$$

Furthermore,

$$
\begin{aligned}
& \left\|x_{n+1}-p\right\|^{2} \\
& \quad=\left\|\gamma_{n} x_{n}+\left(1-\gamma_{n}\right) y_{n}-p\right\|^{2} \\
& \quad \leq \gamma_{n}\left\|x_{n}-p\right\|^{2}+\left(1-\gamma_{n}\right)\left\|y_{n}-p\right\|^{2} \\
& \quad \leq\left\|x_{n}-p\right\|^{2}+\left(1-\gamma_{n}\right)\left(1-\varepsilon_{n} \bar{\gamma}\right)
\end{aligned}
$$




$$
\begin{aligned}
& \times\left(1-\beta_{n}-\varepsilon_{n} \bar{\gamma}\right) \\
& \times\left\{-\alpha_{n} \sum_{i=1}^{N} \delta_{i}\left(\frac{2 v_{i}}{\omega_{i}^{2}}-2 \mu_{i}-\alpha_{n}\right) A_{i}\right. \\
& \times \|\left(\frac{1}{t_{n}} \int_{0}^{t_{n}} T(s) S_{n} u_{n} d s\right. \\
& \left.\left.\quad-\frac{1}{t_{n}} \int_{0}^{t_{n}} T(s) S_{n} p d s\right) \|^{2}\right\} \\
& +\left(1-\gamma_{n}\right) \varepsilon_{n}^{2}\left\|r f\left(x_{n}\right)-A p\right\|^{2} \\
& +2\left(1-\gamma_{n}\right) \varepsilon_{n} \\
& \times\left\langle\left(\left(1-\beta_{n}\right) I-\varepsilon_{n} A\right)\left(z_{n}-p\right),\right. \\
& \left.r f\left(x_{n}\right)-A p\right\rangle \\
& +2\left(1-\gamma_{n}\right) \beta_{n} \varepsilon_{n}\left\langle x_{n}-p, r f\left(x_{n}\right)-A p\right\rangle .
\end{aligned}
$$

It follows that

$$
\begin{aligned}
& \left(1-e_{1}\right)\left(1-\varepsilon_{n} \bar{\gamma}\right)\left(1-\beta_{n}-\varepsilon_{n} \bar{\gamma}\right) \\
& \times\left\{\sum_{i=1}^{N} \delta_{i}\left(\frac{2 g_{1} v_{i}}{\omega_{i}^{2}}-2 g_{2} \mu_{i}-g_{2}^{2}\right)\right. \\
& \times \| A_{i}\left(\frac{1}{t_{n}} \int_{0}^{t_{n}} T(s) S_{n} u_{n} d s\right. \\
& \left.\left.\quad-\frac{1}{t_{n}} \int_{0}^{t_{n}} T(s) S_{n} p d s\right) \|^{2}\right\} \\
& \leq\left(1-\gamma_{n}\right)\left(1-\varepsilon_{n} \bar{\gamma}\right)\left(1-\beta_{n}-\varepsilon_{n} \bar{\gamma}\right) \\
& \times\left\{-\alpha_{n} \sum_{i=1}^{N} \delta_{i}\left(\frac{2 v_{i}}{\omega_{i}^{2}}-2 \mu_{i}-\alpha_{n}\right)\right. \\
& \quad \times \| A_{i}\left(\frac{1}{t_{n}} \int_{0}^{t_{n}} T(s) S_{n} u_{n} d s\right. \\
& \left.\left.\quad-\frac{1}{t_{n}} \int_{0}^{t_{n}} T(s) S_{n} p d s\right) \|^{2}\right\} \\
& \quad\left\|x_{n}-p\right\|^{2}-\left\|x_{n+1}-p\right\|^{2} \\
& +\left(1-\gamma_{n}\right) \varepsilon_{n}^{2}\left\|r f\left(x_{n}\right)-A p\right\|^{2} \\
& +2\left(1-\gamma_{n}\right) \varepsilon_{n} \\
& \left.+2\left(\left(1-\beta_{n}\right) I-\varepsilon_{n} A\right)\left(z_{n}-p\right), r f\left(x_{n}\right)-A p\right\rangle \\
& +2\left(x_{n}-p, r f\left(x_{n}\right)-A p\right\rangle .
\end{aligned}
$$

From (94) and the condition (i), for $i=1,2, \ldots, N$, we have

$$
\lim _{n \rightarrow \infty}\left\|A_{i}\left(\frac{1}{t_{n}} \int_{0}^{t_{n}} T(s) S_{n} u_{n} d s-\frac{1}{t_{n}} \int_{0}^{t_{n}} T(s) S_{n} p d s\right)\right\|=0 .
$$

Then, for $\delta_{i} \in(0,1)$ and $\sum_{i=1}^{N} \delta_{i}=1$,

$$
\begin{aligned}
\lim _{n \rightarrow \infty} \|\left(\sum_{i=1}^{N} \delta_{i} A_{i}\right)( & \frac{1}{t_{n}} \int_{0}^{t_{n}} T(s) S_{n} u_{n} d s \\
& \left.-\frac{1}{t_{n}} \int_{0}^{t_{n}} T(s) S_{n} p d s\right) \|=0 .
\end{aligned}
$$

On the other hand, one has

$\left\|z_{n}-p\right\|^{2}$

$$
\begin{gathered}
=\| P_{C}\left(\frac{1}{t_{n}} \int_{0}^{t_{n}} T(s) S_{n} u_{n} d s\right. \\
\left.\quad-\alpha_{n}\left(\sum_{i=1}^{N} \delta_{i} A_{i}\right) \frac{1}{t_{n}} \int_{0}^{t_{n}} T(s) S_{n} u_{n} d s\right)-p \|^{2} \\
\leq\left\langle\frac{1}{t_{n}} \int_{0}^{t_{n}} T(s) S_{n} u_{n} d s\right. \\
-\alpha_{n}\left(\sum_{i=1}^{N} \delta_{i} A_{i}\right) \frac{1}{t_{n}} \int_{0}^{t_{n}} T(s) S_{n} u_{n} d s \\
-\left(\frac{1}{t_{n}} \int_{0}^{t_{n}} T(s) S_{n} p d s-\alpha_{n}\left(\sum_{i=1}^{N} \delta_{i} A_{i}\right)\right. \\
\left.\left.\quad \times \frac{1}{t_{n}} \int_{0}^{t_{n}} T(s) S_{n} p d s\right), z_{n}-p\right\rangle
\end{gathered}
$$$$
=\frac{1}{2}\left\{\| \frac{1}{t_{n}} \int_{0}^{t_{n}} T(s) S_{n} u_{n} d s\right.
$$

$$
\begin{aligned}
& -\alpha_{n}\left(\sum_{i=1}^{N} \delta_{i} A_{i}\right) \frac{1}{t_{n}} \int_{0}^{t_{n}} T(s) S_{n} u_{n} d s \\
& -\left(\frac{1}{t_{n}} \int_{0}^{t_{n}} T(s) S_{n} p d s\right. \\
& \left.\quad-\alpha_{n}\left(\sum_{i=1}^{N} \delta_{i} A_{i}\right) \frac{1}{t_{n}} \int_{0}^{t_{n}} T(s) S_{n} p d s\right) \|^{2}
\end{aligned}
$$

$+\left\|z_{n}-p\right\|^{2}$

$-\| \frac{1}{t_{n}} \int_{0}^{t_{n}} T(s) S_{n} u_{n} d s$ 
Abstract and Applied Analysis

15

$$
\begin{aligned}
& -\alpha_{n}\left(\sum_{i=1}^{N} \delta_{i} A_{i}\right) \frac{1}{t_{n}} \int_{0}^{t_{n}} T(s) S_{n} u_{n} d s \\
& -\left(\frac{1}{t_{n}} \int_{0}^{t_{n}} T(s) S_{n} p d s-\alpha_{n}\left(\sum_{i=1}^{N} \delta_{i} A_{i}\right)\right. \\
& \left.\left.\times \frac{1}{t_{n}} \int_{0}^{t_{n}} T(s) S_{n} p d s\right)-\left(z_{n}-p\right) \|^{2}\right\} \\
& \leq \frac{1}{2}\left\{\left\|u_{n}-p\right\|^{2}+\left\|z_{n}-p\right\|^{2}\right. \\
& -\|\left(\frac{1}{t_{n}} \int_{0}^{t_{n}} T(s) S_{n} u_{n} d s-z_{n}\right) \\
& -\alpha_{n}\left(\sum_{i=1}^{N} \delta_{i} A_{i}\right) \\
& \times\left(\frac{1}{t_{n}} \int_{0}^{t_{n}} T(s) S_{n} u_{n} d s\right. \\
& \left.\left.-\frac{1}{t_{n}} \int_{0}^{t_{n}} T(s) S_{n} p d s\right) \|^{2}\right\} \\
& \leq \frac{1}{2}\left\{\left\|x_{n}-p\right\|^{2}+\left\|z_{n}-p\right\|^{2}\right. \\
& -\left\|\frac{1}{t_{n}} \int_{0}^{t_{n}} T(s) S_{n} u_{n} d s-z_{n}\right\|^{2} \\
& -\alpha_{n}^{2} \|\left(\sum_{i=1}^{N} \delta_{i} A_{i}\right) \\
& \times\left(\frac{1}{t_{n}} \int_{0}^{t_{n}} T(s) S_{n} u_{n} d s-\frac{1}{t_{n}} \int_{0}^{t_{n}} T(s) S_{n} p d s\right) \|^{2} \\
& +2 \alpha_{n}\left\langle\left(\sum_{i=1}^{N} \delta_{i} A_{i}\right)\right. \\
& \times\left(\frac{1}{t_{n}} \int_{0}^{t_{n}} T(s) S_{n} u_{n} d s-\frac{1}{t_{n}} \int_{0}^{t_{n}} T(s) S_{n} p d s\right), \\
& \left.\left.\frac{1}{t_{n}} \int_{0}^{t_{n}} T(s) S_{n} u_{n} d s-z_{n}\right\rangle\right\} \text {, }
\end{aligned}
$$

which means that

$$
\begin{aligned}
\left\|z_{n}-p\right\|^{2} \leq & \left\|x_{n}-p\right\|^{2}-\left\|\frac{1}{t_{n}} \int_{0}^{t_{n}} T(s) S_{n} u_{n} d s-z_{n}\right\|^{2} \\
& +2 \alpha_{n} \|\left(\sum_{i=1}^{N} \delta_{i} A_{i}\right)
\end{aligned}
$$

$$
\begin{gathered}
\times\left(\frac{1}{t_{n}} \int_{0}^{t_{n}} T(s) S_{n} u_{n} d s\right. \\
\left.-\frac{1}{t_{n}} \int_{0}^{t_{n}} T(s) S_{n} p d s\right) \| \\
\times\left\|\frac{1}{t_{n}} \int_{0}^{t_{n}} T(s) S_{n} u_{n} d s-z_{n}\right\| .
\end{gathered}
$$

It follows that

$$
\begin{aligned}
& \left\|y_{n}-p\right\|^{2} \\
& \leq\left(1-\varepsilon_{n} \bar{\gamma}\right)\left(1-\beta_{n}-\varepsilon_{n} \bar{\gamma}\right)\left\|z_{n}-p\right\|^{2} \\
& +\left(1-\varepsilon_{n} \bar{\gamma}\right) \beta_{n}\left\|x_{n}-p\right\|^{2} \\
& +\varepsilon_{n}^{2}\left\|r f\left(x_{n}\right)-A p\right\|^{2} \\
& +2 \varepsilon_{n}\left\langle\left(\left(1-\beta_{n}\right) I-\varepsilon_{n} A\right)\left(z_{n}-p\right),\right. \\
& \left.r f\left(x_{n}\right)-A p\right\rangle \\
& +2 \beta_{n} \varepsilon_{n}\left\langle x_{n}-p, r f\left(x_{n}\right)-A p\right\rangle \\
& \leq\left(1-\varepsilon_{n} \bar{\gamma}\right)\left(1-\beta_{n}-\varepsilon_{n} \bar{\gamma}\right) \\
& \times\left\{\left\|x_{n}-p\right\|^{2}-\left\|\frac{1}{t_{n}} \int_{0}^{t_{n}} T(s) S_{n} u_{n} d s-z_{n}\right\|^{2}\right. \\
& +2 \alpha_{n} \|\left(\sum_{i=1}^{N} \delta_{i} A_{i}\right) \\
& \times\left(\frac{1}{t_{n}} \int_{0}^{t_{n}} T(s) S_{n} u_{n} d s\right. \\
& \left.-\frac{1}{t_{n}} \int_{0}^{t_{n}} T(s) S_{n} p d s\right) \| \\
& \left.\times\left\|S_{n} u_{n}-z_{n}\right\|\right\} \\
& +\left(1-\varepsilon_{n} \bar{\gamma}\right) \beta_{n}\left\|x_{n}-p\right\|^{2}+\varepsilon_{n}^{2}\left\|r f\left(x_{n}\right)-A p\right\|^{2} \\
& +2 \varepsilon_{n}\left\langle\left(\left(1-\beta_{n}\right) I-\varepsilon_{n} A\right)\left(z_{n}-p\right), r f\left(x_{n}\right)-A p\right\rangle \\
& +2 \beta_{n} \varepsilon_{n}\left\langle x_{n}-p, r f\left(x_{n}\right)-A p\right\rangle \\
& =\left(1-\varepsilon_{n} \bar{\gamma}\right)^{2}\left\|x_{n}-p\right\|^{2}-\left(1-\varepsilon_{n} \bar{\gamma}\right) \\
& \times\left(1-\beta_{n}-\varepsilon_{n} \bar{\gamma}\right)\left\|\frac{1}{t_{n}} \int_{0}^{t_{n}} T(s) S_{n} u_{n} d s-z_{n}\right\|^{2} \\
& +2 \alpha_{n}\left(1-\varepsilon_{n} \bar{\gamma}\right)\left(1-\beta_{n}-\varepsilon_{n} \bar{\gamma}\right)
\end{aligned}
$$


16

Abstract and Applied Analysis

$$
\begin{aligned}
& \times \|\left(\sum_{i=1}^{N} \delta_{i} A_{i}\right) \\
& \times\left(\frac{1}{t_{n}} \int_{0}^{t_{n}} T(s) S_{n} u_{n} d s\right. \\
& \left.\quad-\frac{1}{t_{n}} \int_{0}^{t_{n}} T(s) S_{n} p d s\right)\|\| S_{n} u_{n}-z_{n} \| \\
& +\varepsilon_{n}^{2}\left\|r f\left(x_{n}\right)-A p\right\|^{2} \\
& +2 \varepsilon_{n}\left\langle\left(\left(1-\beta_{n}\right) I-\varepsilon_{n} A\right)\left(z_{n}-p\right),\right. \\
& +2 \beta_{n} \varepsilon_{n}\left\langle x_{n}-p, r f\left(x_{n}\right)-A p\right\rangle \\
& \leq\left\|x_{n}-p\right\|^{2}-\left(1-\varepsilon_{n} \bar{\gamma}\right)\left(1-\beta_{n}-\varepsilon_{n} \bar{\gamma}\right) \\
& \times\left\|\frac{1}{t_{n}} \int_{0}^{t_{n}} T(s) S_{n} u_{n} d s-z_{n}\right\|^{2} \\
& +2 \alpha_{n}\left(1-\varepsilon_{n} \bar{\gamma}\right)\left(1-\beta_{n}-\varepsilon_{n} \bar{\gamma}\right) \\
& +\|\left(\sum_{n}^{2}\left\|r f\left(x_{n}\right)-A p\right\|^{2}+2 \varepsilon_{n} \delta_{n} \delta_{i} A_{i}\right)\left(\frac{1}{t_{n}} \int_{0}^{t_{n}} T(s) S_{n} u_{n} d s\right. \\
& \times \\
& +2 \beta_{n} \varepsilon_{n}\left\langle x_{n}-p, r f\left(x_{n}\right)-A p\right\rangle .
\end{aligned}
$$

(108)

Therefore, from (108) and (102), one has

$$
\begin{aligned}
& \left\|x_{n+1}-p\right\|^{2} \\
& \leq \gamma_{n}\left\|x_{n}-p\right\|^{2}+\left(1-\gamma_{n}\right)\left\|y_{n}-p\right\|^{2} \\
& \leq \gamma_{n}\left\|x_{n}-p\right\|^{2}+\left(1-\gamma_{n}\right) \\
& \times\left\{\left\|x_{n}-p\right\|^{2}-\left(1-\varepsilon_{n} \bar{\gamma}\right)\right. \\
& \times\left(1-\beta_{n}-\varepsilon_{n} \bar{\gamma}\right) \\
& \left.\times\left\|\frac{1}{t_{n}} \int_{0}^{t_{n}} T(s) S_{n} u_{n} d s-z_{n}\right\|^{2}\right\} \\
& +2 \alpha_{n}\left(1-\varepsilon_{n} \bar{\gamma}\right)\left(1-\beta_{n}-\varepsilon_{n} \bar{\gamma}\right) \\
& \times\left\{\|\left(\sum_{i=1}^{N} \delta_{i} A_{i}\right)\right. \\
& \times\left(\frac{1}{t_{n}} \int_{0}^{t_{n}} T(s) S_{n} u_{n} d s\right. \\
& \left.-\frac{1}{t_{n}} \int_{0}^{t_{n}} T(s) S_{n} p d s\right) \|
\end{aligned}
$$

$$
\begin{aligned}
& \times\left\|S_{n} u_{n}-z_{n}\right\|+\varepsilon_{n}^{2}\left\|r f\left(x_{n}\right)-A p\right\|^{2} \\
& +2 \varepsilon_{n}\left\langle\left(\left(1-\beta_{n}\right) I-\varepsilon_{n} A\right)\left(z_{n}-p\right)\right. \text {, } \\
& \left.r f\left(x_{n}\right)-A p\right\rangle \\
& \left.+2 \beta_{n} \varepsilon_{n}\left\langle x_{n}-p, r f\left(x_{n}\right)-A p\right\rangle\right\} \\
& =\left\|x_{n}-p\right\|^{2}-\left(1-\gamma_{n}\right)\left(1-\varepsilon_{n} \bar{\gamma}\right) \\
& \times\left(1-\beta_{n}-\varepsilon_{n} \bar{\gamma}\right)\left\|\frac{1}{t_{n}} \int_{0}^{t_{n}} T(s) S_{n} u_{n} d s-z_{n}\right\|^{2} \\
& +2 \alpha_{n}\left(1-\gamma_{n}\right)\left(1-\varepsilon_{n} \bar{\gamma}\right)\left(1-\beta_{n}-\varepsilon_{n} \bar{\gamma}\right) \\
& \times\left\{\|\left(\sum_{i=1}^{N} \delta_{i} A_{i}\right)\right. \\
& \times\left(\frac{1}{t_{n}} \int_{0}^{t_{n}} T(s) S_{n} u_{n} d s\right. \\
& \left.-\frac{1}{t_{n}} \int_{0}^{t_{n}} T(s) S_{n} p d s\right) \| \\
& \times\left\|S_{n} u_{n}-z_{n}\right\|+\left(1-\gamma_{n}\right) \varepsilon_{n}^{2} \\
& \times\left\|r f\left(x_{n}\right)-A p\right\|^{2}+2\left(1-\gamma_{n}\right) \varepsilon_{n} \\
& \times\left\langle\left(\left(1-\beta_{n}\right) I-\varepsilon_{n} A\right)\left(z_{n}-p\right),\right. \\
& \left.r f\left(x_{n}\right)-A p\right\rangle \\
& +2\left(1-\gamma_{n}\right) \beta_{n} \varepsilon_{n} \\
& \left.\times\left\langle x_{n}-p, r f\left(x_{n}\right)-A p\right\rangle\right\} \text {. }
\end{aligned}
$$

Then,

$$
\begin{aligned}
& \left(1-\gamma_{n}\right)\left(1-\varepsilon_{n} \bar{\gamma}\right)\left(1-\beta_{n}-\varepsilon_{n} \bar{\gamma}\right) \\
& \quad \times\left\|\frac{1}{t_{n}} \int_{0}^{t_{n}} T(s) S_{n} u_{n} d s-z_{n}\right\|^{2} \\
& \leq\left\|x_{n}-p\right\|^{2}-\left\|x_{n+1}-p\right\|^{2} \\
& +2 \alpha_{n}\left(1-\gamma_{n}\right)\left(1-\varepsilon_{n} \bar{\gamma}\right)\left(1-\beta_{n}-\varepsilon_{n} \bar{\gamma}\right) \\
& \quad \times\left\{\|\left(\sum_{i=1}^{N} \delta_{i} A_{i}\right)\left(\frac{1}{t_{n}} \int_{0}^{t_{n}} T(s) S_{n} u_{n} d s\right.\right. \\
& \left.\quad-\frac{1}{t_{n}} \int_{0}^{t_{n}} T(s) S_{n} p d s\right) \| \\
& \times\left\|S_{n} u_{n}-z_{n}\right\|+\left(1-\gamma_{n}\right) \varepsilon_{n}^{2}
\end{aligned}
$$




$$
\begin{aligned}
& \times\left\|r f\left(x_{n}\right)-A p\right\|^{2}+2\left(1-\gamma_{n}\right) \varepsilon_{n} \\
& \times\left\langle\left(\left(1-\beta_{n}\right) I-\varepsilon_{n} A\right)\left(z_{n}-p\right), r f\left(x_{n}\right)-A p\right\rangle \\
& +2\left(1-\gamma_{n}\right) \beta_{n} \varepsilon_{n} \\
& \left.\times\left\langle x_{n}-p, r f\left(x_{n}\right)-A p\right\rangle\right\} .
\end{aligned}
$$

From (94), (105), and condition (i), one has

$$
\lim _{n \rightarrow \infty}\left\|\frac{1}{t_{n}} \int_{0}^{t_{n}} T(s) S_{n} u_{n} d s-z_{n}\right\|=0 .
$$

Let $p \in \Theta$ and $k \in\{1,2, \ldots, M\}$. Since $J_{r_{k, n}}^{F_{k}}$ is firmly nonexpansive, we obtain

$$
\begin{aligned}
& \left\|\Im_{n}^{k} x_{n}-p\right\|^{2} \\
& =\left\|J_{r_{k, n}}^{F_{k}} \Im_{n}^{k-1} x_{n}-J_{r_{k, n}}^{F_{k}} p\right\|^{2} \\
& =\left\langle J_{r_{k, n}}^{F_{k}} \Im_{n}^{k-1} x_{n}-p, \Im_{n}^{k-1} x_{n}-p\right\rangle \\
& =\frac{1}{2}\left(\left\|\Im_{n}^{k} x_{n}-p\right\|^{2}+\left\|\Im_{n}^{k-1} x_{n}-p\right\|^{2}\right. \\
& \left.\quad-\left\|\Im_{n}^{k} x_{n}-\Im_{n}^{k-1} x_{n}\right\|^{2}\right) .
\end{aligned}
$$

It follows that

$$
\left\|\mathfrak{\Im}_{n}^{k} x_{n}-p\right\|^{2} \leq\left\|x_{n}-p\right\|^{2}-\left\|\mathfrak{\Im}_{n}^{k} x_{n}-\mathfrak{\Im}_{n}^{k-1} x_{n}\right\|^{2}
$$

Consequently, from (108), one has

$$
\begin{aligned}
\| y_{n}- & p \|^{2} \\
\leq & \left(1-\varepsilon_{n} \bar{\gamma}\right)\left(1-\beta_{n}-\varepsilon_{n} \bar{\gamma}\right)\left\|z_{n}-p\right\|^{2} \\
& +\left(1-\varepsilon_{n} \bar{\gamma}\right) \beta_{n}\left\|x_{n}-p\right\|^{2} \\
& +\varepsilon_{n}^{2}\left\|r f\left(x_{n}\right)-A p\right\|^{2}+2 \varepsilon_{n} \\
& \times\left\langle\left(\left(1-\beta_{n}\right) I-\varepsilon_{n} A\right)\left(z_{n}-p\right), r f\left(x_{n}\right)-A p\right\rangle \\
& +2 \beta_{n} \varepsilon_{n}\left\langle x_{n}-p, r f\left(x_{n}\right)-A p\right\rangle \\
\leq & \left(1-\varepsilon_{n} \bar{\gamma}\right)\left(1-\beta_{n}-\varepsilon_{n} \bar{\gamma}\right) \\
& \times\left\|u_{n}-p\right\|^{2}+\left(1-\varepsilon_{n} \bar{\gamma}\right) \beta_{n}\left\|x_{n}-p\right\|^{2} \\
& +\varepsilon_{n}^{2}\left\|r f\left(x_{n}\right)-A p\right\|^{2}+2 \varepsilon_{n} \\
& \times\left\langle\left(\left(1-\beta_{n}\right) I-\varepsilon_{n} A\right)\left(z_{n}-p\right),\right. \\
& \left.\quad r f\left(x_{n}\right)-A p\right\rangle
\end{aligned}
$$

$$
\begin{aligned}
& +2 \beta_{n} \varepsilon_{n}\left\langle x_{n}-p, r f\left(x_{n}\right)-A p\right\rangle \\
& =\left(1-\varepsilon_{n} \bar{\gamma}\right)\left(1-\beta_{n}-\varepsilon_{n} \bar{\gamma}\right) \\
& \times\left\|\mathfrak{J}_{n}^{k} x_{n}-p\right\|^{2}+\left(1-\varepsilon_{n} \bar{\gamma}\right) \beta_{n} \\
& \times\left\|x_{n}-p\right\|^{2}+\varepsilon_{n}^{2}\left\|r f\left(x_{n}\right)-A p\right\|^{2} \\
& +2 \varepsilon_{n}\left\langle\left(\left(1-\beta_{n}\right) I-\varepsilon_{n} A\right)\left(z_{n}-p\right),\right. \\
& \left.r f\left(x_{n}\right)-A p\right\rangle \\
& +2 \beta_{n} \varepsilon_{n}\left\langle x_{n}-p, r f\left(x_{n}\right)-A p\right\rangle \\
& \leq\left(1-\varepsilon_{n} \bar{\gamma}\right)\left(1-\beta_{n}-\varepsilon_{n} \bar{\gamma}\right) \\
& \times\left(\left\|x_{n}-p\right\|^{2}-\left\|\Im_{n}^{k} x_{n}-\Im_{n}^{k-1} x_{n}\right\|^{2}\right) \\
& +\left(1-\varepsilon_{n} \bar{\gamma}\right) \beta_{n}\left\|x_{n}-p\right\|^{2} \\
& +\varepsilon_{n}^{2}\left\|r f\left(x_{n}\right)-A p\right\|^{2} \\
& +2 \varepsilon_{n}\left\langle\left(\left(1-\beta_{n}\right) I-\varepsilon_{n} A\right)\left(z_{n}-p\right),\right. \\
& \left.r f\left(x_{n}\right)-A p\right\rangle \\
& +2 \beta_{n} \varepsilon_{n}\left\langle x_{n}-p, r f\left(x_{n}\right)-A p\right\rangle \\
& \leq\left\|x_{n}-p\right\|^{2}-\left(1-\varepsilon_{n} \bar{\gamma}\right)\left(1-\beta_{n}-\varepsilon_{n} \bar{\gamma}\right) \\
& \times\left\|\Im_{n}^{k} x_{n}-\Im_{n}^{k-1} x_{n}\right\|^{2}+\varepsilon_{n}^{2}\left\|r f\left(x_{n}\right)-A p\right\|^{2} \\
& +2 \varepsilon_{n}\left\langle\left(\left(1-\beta_{n}\right) I-\varepsilon_{n} A\right)\left(z_{n}-p\right),\right. \\
& \left.r f\left(x_{n}\right)-A p\right\rangle \\
& +2 \beta_{n} \varepsilon_{n}\left\langle x_{n}-p, r f\left(x_{n}\right)-A p\right\rangle \text {. }
\end{aligned}
$$

Then,

$$
\begin{aligned}
& \left\|x_{n+1}-p\right\|^{2} \\
& =\left\|\gamma_{n} x_{n}+\left(1-\gamma_{n}\right) y_{n}-p\right\|^{2} \\
& \leq \gamma_{n}\left\|x_{n}-p\right\|^{2}+\left(1-\gamma_{n}\right)\left\|y_{n}-p\right\|^{2} \\
& \leq\left\|x_{n}-p\right\|^{2}+\left(1-\gamma_{n}\right) \\
& \times\left\{-\left(1-\varepsilon_{n} \bar{\gamma}\right)\left(1-\beta_{n}-\varepsilon_{n} \bar{\gamma}\right)\right. \\
& \quad \times\left\|\Im_{n}^{k} x_{n}-\Im_{n}^{k-1} x_{n}\right\|^{2} \\
& +\varepsilon_{n}^{2}\left\|r f\left(x_{n}\right)-A p\right\|^{2} \\
& +2 \varepsilon_{n}\left\langle\left(\left(1-\beta_{n}\right) I-\varepsilon_{n} A\right)\left(z_{n}-p\right),\right. \\
& \left.\quad r f\left(x_{n}\right)-A p\right\rangle \\
& \left.+2 \beta_{n} \varepsilon_{n}\left\langle x_{n}-p, r f\left(x_{n}\right)-A p\right\rangle\right\} .
\end{aligned}
$$


That is,

$$
\begin{aligned}
&\left(1-\gamma_{n}\right)-\left(1-\varepsilon_{n} \bar{\gamma}\right)\left(1-\beta_{n}-\varepsilon_{n} \bar{\gamma}\right) \\
& \quad \times\left\|\Im_{n}^{k} x_{n}-\Im_{n}^{k-1} x_{n}\right\|^{2} \\
& \leq\left\|x_{n}-p\right\|^{2}-\left\|x_{n+1}-p\right\|^{2} \\
&+\left(1-\gamma_{n}\right) \varepsilon_{n}^{2}\left\|r f\left(x_{n}\right)-A p\right\|^{2} \\
&+2\left(1-\gamma_{n}\right) \varepsilon_{n} \\
& \times\left\langle\left(\left(1-\beta_{n}\right) I-\varepsilon_{n} A\right)\left(z_{n}-p\right), r f\left(x_{n}\right)-A p\right\rangle \\
&+2\left(1-\gamma_{n}\right) \beta_{n} \varepsilon_{n}\left\langle x_{n}-p, r f\left(x_{n}\right)-A p\right\rangle .
\end{aligned}
$$

By condition (i) and (94), for $k \in\{1,2, \ldots, M\}$, we obtain

$$
\lim _{n \rightarrow \infty}\left\|\mathfrak{\Im}_{n}^{k} x_{n}-\mathfrak{\Im}_{n}^{k-1} x_{n}\right\|=0 .
$$

Therefore, we have

$$
\begin{aligned}
\| u_{n}- & x_{n} \| \\
= & \left\|\Im_{n}^{k} x_{n}-\Im_{n}^{0} x_{n}\right\| \leq\left\|\Im_{n}^{k} x_{n}-\Im_{n}^{k-1} x_{n}\right\| \\
& +\left\|\Im_{n}^{k-1} x_{n}-\Im_{n}^{k-2} x_{n}\right\|+\cdots+\left\|\Im_{n}^{1} x_{n}-\Im_{n}^{0} x_{n}\right\| .
\end{aligned}
$$

From (117), one has

$$
\lim _{n \rightarrow \infty}\left\|u_{n}-x_{n}\right\|=0
$$

Notice that

$$
\left\|u_{n}-y_{n}\right\| \leq\left\|u_{n}-x_{n}\right\|+\left\|x_{n}-y_{n}\right\|,
$$

Applying (119) and (93), we have

$$
\lim _{n \rightarrow \infty}\left\|u_{n}-y_{n}\right\|=0 .
$$

Since

$$
\left\|u_{n}-z_{n}\right\| \leq\left\|u_{n}-y_{n}\right\|+\left\|y_{n}-z_{n}\right\|,
$$

this together with (94) yields that

$$
\lim _{n \rightarrow \infty}\left\|u_{n}-z_{n}\right\|=0
$$

Consequently, we obtain

$$
\lim _{n \rightarrow \infty}\left\|\frac{1}{t_{n}} \int_{0}^{t_{n}} T(s) S_{n} u_{n} d s-u_{n}\right\|=0 .
$$

Step 4. Letting $z=P_{\Theta}(I-A+r f) z$, we show

$$
\lim _{n \rightarrow \infty} \sup \left\langle(r f-A) z, x_{n}-z\right\rangle \leq 0 .
$$

We know that $P_{\Theta}(I-A+r f)$ is a contraction. Indeed, for any $x, y \in H$, we have

$$
\begin{aligned}
\| P_{\Theta}( & I-A+r f) x-P_{\Theta}(I-A+r f) y \| \\
& \leq\|(I-A+r f) x-(I-A+r f) y\| \\
& \leq(1-(\bar{\gamma}-r \eta))\|x-y\|,
\end{aligned}
$$

and hence $P_{\Theta}(I-A+r f)$ is a contraction due to $(1-(\bar{\gamma}-$ $r \eta)) \in(0,1)$. Thus, Banach's Contraction Mapping Principle guarantees that $P_{\Theta}(I-A+r f)$ has a unique fixed point, which implies $z=P_{\Theta}(I-A+r f) z$.

We claim that $z \in F(\Gamma)$. Since $\left\{u_{n_{i}}\right\} \subset\left\{u_{n}\right\}$ is bounded in $C$, without loss of generality, we can assume that $\left\{u_{n_{i}}\right\} \rightarrow z$. Since $C$ is closed and convex, $C$ is weakly closed. Thus we have $z \in C$. For $0 \leq s<\infty$, notice that

$$
\begin{aligned}
& \left\|u_{n_{i}}-T(h) u_{n_{i}}\right\| \\
& \leq\left\|u_{n_{i}}-\frac{1}{t_{n_{i}}} \int_{0}^{t_{n_{i}}} T(s) S_{n_{i}} u_{n_{i}} d s\right\| \\
& +\| \frac{1}{t_{n_{i}}} \int_{0}^{t_{n_{i}}} T(s) S_{n_{i}} u_{n_{i}} d s \\
& +\quad\left\|T(h) \frac{1}{t_{n_{i}}} \int_{0}^{t_{n_{i}}} T(s) S_{n_{i}} u_{n_{i}} d s\right\| \\
& \leq 2\left\|u_{n_{i}}-\frac{1}{t_{n_{i}}} \int_{0}^{t_{n_{i}}} T(s) S_{n_{i}} u_{n_{i}} d s-T(h) u_{n_{i}}\right\| \\
& +\left\|\frac{1}{t_{n_{i}}} T(s) S_{n_{i}} u_{n_{i}} d s\right\| \\
& \quad \int_{n_{n_{i}}}^{t_{n_{i}}} T(s) S_{n_{i}} u_{n_{i}} d s \\
& \quad-T(h) \frac{1}{t_{n_{i}}} \int_{0}^{t_{n_{i}}} T(s) S_{n_{i}} u_{n_{i}} d s \| .
\end{aligned}
$$

It follows from (124) and Lemma 4 that

$$
\lim _{n \rightarrow \infty}\left\|u_{n_{i}}-T(h) u_{n_{i}}\right\|=0 .
$$

Thus, (128) and Lemma 5 assert that $z \in F(\Gamma)$. Since $\left\{x_{n_{i}}\right\} \subset$ $\left\{x_{n}\right\}$ is bounded in $C$, without loss of generality, we can assume that $\left\{x_{n_{i}}\right\} \rightarrow \omega$. It follows from (94) that $z_{n_{i}} \rightarrow \omega$. Since $C$ is closed and convex, $C$ is weakly closed. Thus we have $\omega \in C$.

Let us show $\omega \in F(S)$. For the sake of contradiction, suppose that $\omega \notin F(S)$, that is, $S \omega \neq \omega$. Since $z \in F(\Gamma)$, by our assumption, we have $T_{i} \omega \in F(\Gamma)$ and then $S_{n} \omega \in F(\Gamma)$. Hence $\left(1 / t_{n}\right) \int_{0}^{t_{n}} T(s) S_{n} \omega d s=S_{n} \omega$. Therefore, by (124) and Opial condition, we have

$$
\begin{aligned}
& \lim _{n \rightarrow \infty} \inf \left\|u_{n_{i}}-\omega\right\| \\
& \quad<\lim _{n \rightarrow \infty} \inf \left\|u_{n_{i}}-S \omega\right\| \\
& \quad \leq \lim _{n \rightarrow \infty} \inf \left\{\left\|u_{n_{i}}-\frac{1}{t_{n_{i}}} \int_{0}^{t_{n_{i}}} T(s) S_{n_{i}} u_{n_{i}} d s\right\|\right.
\end{aligned}
$$




$$
\begin{gathered}
+\| \frac{1}{t_{n_{i}}} \int_{0}^{t_{n_{i}}} T(s) S_{n_{i}} u_{n_{i}} d s \\
\quad-\frac{1}{t_{n_{i}}} \int_{0}^{t_{n_{i}}} T(s) S_{n_{i}} \omega d s \| \\
\left.+\left\|S_{n_{i}} \omega-S \omega\right\|\right\} \\
\leq \lim _{n \rightarrow \infty} \inf \left\|u_{n_{i}}-\omega\right\|,
\end{gathered}
$$

which derives a contradiction. Thus, we obtain $\omega \in F(S)=$ $\cap_{i=1}^{\infty} F\left(T_{i}\right)$

Next, we claim that $\omega \in \cap_{i=1}^{M} \operatorname{SEP}\left(F_{i}\right)$. Since $u_{n}=\Im_{n}^{k} x_{n}$ for $k=1,2, \ldots, M$, we obtain

$$
\begin{aligned}
& F_{k}\left(\Im_{n}^{k} x_{n}, y\right) \\
& \quad+\frac{1}{r_{n}}\left\langle y-\Im_{n}^{k} x_{n}, \Im_{n}^{k} x_{n}-\Im_{n}^{k-1} x_{n}\right\rangle \geq 0, \quad \forall y \in C .
\end{aligned}
$$

From (A2), one has

$$
\frac{1}{r_{n}}\left\langle y-\mathfrak{\Im}_{n}^{k} x_{n}, \Im_{n}^{k} x_{n}-\Im_{n}^{k-1} x_{n}\right\rangle \geq F\left(y, \Im_{n}^{k} x_{n}\right)
$$

Replacing $n$ by $n_{i}$, we have

$$
\left\langle y-\Im_{n_{i}}^{k} x_{n_{i}}, \frac{1}{r_{n_{i}}}\left(\Im_{n_{i}}^{k} x_{n_{i}}-\Im_{n_{i}}^{k-1} x_{n_{i}}\right)\right\rangle \geq F_{k}\left(y, \Im_{n_{i}}^{k} x_{n_{i}}\right) .
$$

It follows from $\left(1 / r_{n_{i}}\right)\left(\Im_{n_{i}}^{k} x_{n_{i}}-\Im_{n_{i}}^{k-1} x_{n_{i}}\right) \rightarrow 0$ and $\Im_{n_{i}}^{k} x_{n_{i}} \rightarrow$ $\omega$ that

$$
F_{k}(y, \omega) \leq 0, \quad y \in C,
$$

for $k=1,2, \ldots, M$.

Put $z_{t}=t y+(1-t) \omega$ for all $t \in(0,1]$ and $y \in C$. Then, we have $z_{t} \in C$ and then $F\left(z_{t}, \omega\right) \leq 0$. Hence, from (A1) and (A4), we have

$$
\begin{aligned}
0 & =F_{k}\left(z_{t}, z_{t}\right) \leq t F_{k}\left(z_{t}, y\right)+(1-t) F_{k}\left(z_{t}, y\right) \\
& \leq t F_{k}\left(z_{t}, y\right),
\end{aligned}
$$

which means $F_{k}\left(z_{t}, y\right) \geq 0$. From (A3), we obtain $F_{k}(\omega, y) \geq 0$ for $y \in C$ and then $\omega \in \operatorname{SEP}\left(F_{k}\right)$ for $k=1,2, \ldots, M$, that is, $\omega \in \cap_{i=1}^{M} \operatorname{SEP}\left(F_{k}\right)$.

Finally, we claim that $\omega \in \cap_{i=1}^{N} \operatorname{VI}\left(C, A_{i}\right)$.

We define the maximal monotone operator

$$
Q q_{1}= \begin{cases}\left(\sum_{i=1}^{N} \delta_{i} A_{i}\right) q_{1}+N_{C} q_{1}, & \omega_{1} \in C, \\ \emptyset, & \omega_{1} \notin C .\end{cases}
$$

Since $A_{i}$ is relaxed $\left(\mu_{i}, v_{i}\right)$-cocoercive for $i=1,2$, we have

$$
\begin{aligned}
& \left\langle\left(\sum_{i=1}^{N} \delta_{i} A_{i}\right) x-\left(\sum_{i=1}^{N} \delta_{i} A_{i}\right) y, x-y\right\rangle \\
& =\sum_{i=1}^{N} \delta_{i}\left\langle A_{i} x-A_{i} y, x-y\right\rangle \\
& \geq \sum_{i=1}^{N} \delta_{i}\left(-\mu_{i}\left\|A_{i} x-A_{i} y\right\|^{2}+v_{i}\|x-y\|^{2}\right) \\
& \geq \sum_{i=1}^{N} \delta_{i}\left(v_{i}-\mu_{i} \omega_{1}^{i}\right)\|x-y\|^{2} \\
& \geq 0
\end{aligned}
$$

which yields that $\sum_{i=1}^{N} \delta_{i} A_{i}$ is monotone. Thus, $Q$ is maximal monotone. Let $\left(q_{1}, q_{2}\right) \in G(Q)$. Since $q_{2}-\left(\sum_{i=1}^{N} \delta_{i} A_{i}\right) q_{1} \in$ $N_{C} q_{1}$ and $z_{n} \in C$, we have

$$
\left\langle q_{1}-z_{n}, q_{2}-\left(\sum_{i=1}^{N} \delta_{i} A_{i}\right) q_{1}\right\rangle \geq 0
$$

On the other hand, it follows from $z_{n}=P_{C}\left(\left(1 / t_{n}\right)\right.$ $\left.\int_{0}^{t_{n}} T(s) S_{n} u_{n} d s-\alpha_{n}\left(\sum_{i=1}^{N} \delta_{i} A_{i}\right)\left(1 / t_{n}\right) \int_{0}^{t_{n}} T(s) S_{n} u_{n} d s\right)$ that

$$
\begin{aligned}
& \left\langle q_{1}-z_{n}, z_{n}\right. \\
& -\left(\frac{1}{t_{n}} \int_{0}^{t_{n}} T(s) S_{n} u_{n} d s-\alpha_{n}\left(\sum_{i=1}^{N} \delta_{i} A_{i}\right)\right. \\
& \left.\left.\times \frac{1}{t_{n}} \int_{0}^{t_{n}} T(s) S_{n} u_{n} d s\right)\right\rangle \geq 0,
\end{aligned}
$$

and hence

$$
\begin{aligned}
& \left\langle q_{1}-z_{n}, \frac{z_{n}-\left(1 / t_{n}\right) \int_{0}^{t_{n}} T(s) S_{n} u_{n} d s}{\alpha_{n}}\right. \\
& \left.+\left(\sum_{i=1}^{N} \delta_{i} A_{i}\right) \frac{1}{t_{n}} \int_{0}^{t_{n}} T(s) S_{n} u_{n} d s\right\rangle \geq 0 .
\end{aligned}
$$


It follows that

$$
\begin{aligned}
& \left\langle q_{1}-z_{n_{i}}, q_{2}\right\rangle \\
& \geq\left\langle q_{1}-z_{n_{i}},\left(\sum_{i=1}^{N} \delta_{i} A_{i}\right) q_{1}\right\rangle \\
& \geq\left\langle q_{1}-z_{n_{i}},\left(\sum_{i=1}^{N} \delta_{i} A_{i}\right) q_{1}\right\rangle \\
& -\left\langle q_{1}-z_{n_{i}}, \frac{z_{n_{i}}-S_{n_{i}} u_{n_{i}}}{\alpha_{n_{i}}}\right. \\
& \left.+\left(\sum_{i=1}^{N} \delta_{i} A_{i}\right) S_{n_{i}} u_{n_{i}}\right\rangle \\
& =\left\langle q_{1}-z_{n_{i}},\left(\sum_{i=1}^{N} \delta_{i} A_{i}\right)\right. \\
& \times\left(q_{1}-\frac{1}{t_{n_{i}}} \int_{0}^{t_{n_{i}}} T(s) S_{n_{i}} u_{n_{i}} d s\right) \\
& \left.-\frac{z_{n_{i}}-\left(1 / t_{n_{i}}\right) \int_{0}^{t_{n_{i}}} T(s) S_{n_{i}} u_{n_{i}} d s}{\alpha_{n_{i}}}\right\rangle \\
& =\left\langle q_{1}-z_{n_{i}},\left(\sum_{i=1}^{N} \delta_{i} A_{i}\right)\left(q_{1}-z_{n_{i}}\right)\right\rangle \\
& +\left\langle q_{1}-z_{n_{i}},\left(\sum_{i=1}^{N} \delta_{i} A_{i}\right)\left(z_{n_{i}}-S_{n_{i}} u_{n_{i}}\right)\right\rangle \\
& -\left\langle q_{1}-z_{n_{i}}, \frac{z_{n_{i}}-S_{n_{i}} u_{n_{i}}}{\alpha_{n_{i}}}\right\rangle \\
& \geq\left\langle q_{1}-z_{n_{i}},\left(\sum_{i=1}^{N} \delta_{i} A_{i}\right)\right. \\
& \left.\times\left(z_{n_{i}}-\frac{1}{t_{n_{i}}} \int_{0}^{t_{n_{i}}} T(s) S_{n_{i}} u_{n_{i}} d s\right)\right\rangle \\
& -\left\langle q_{1}-z_{n_{i}}, \frac{z_{n_{i}}-\left(1 / t_{n_{i}}\right) \int_{0}^{t_{n_{i}}} T(s) S_{n_{i}} u_{n_{i}} d s}{\alpha_{n_{i}}}\right\rangle,
\end{aligned}
$$

which implies that

$$
\left\langle q_{1}-\omega, q_{2}\right\rangle \geq 0
$$

Since $Q$ is maximal monotone, we obtain that $\omega \in Q^{-1} 0$. From Lemma 8, we obtain $\omega \in \operatorname{VI}\left(C, \sum_{i=1}^{N} \delta_{i} A_{i}\right)$, that is, $\omega \in\left(\cap_{i=1}^{N} \operatorname{VI}\left(C, A_{i}\right)\right)$. Thus, $\omega \in \Theta$.
Since $z=P_{\Theta}(I-A+r f) z$, one has

$$
\begin{aligned}
& \lim _{n \rightarrow \infty} \sup \left\langle(r f-A) z, x_{n}-z\right\rangle \\
& \quad=\lim _{i \rightarrow \infty}\left\langle(r f-A) z, x_{n_{i}}-z\right\rangle \\
& \quad=\langle(r f-A) z, \omega-z\rangle \\
& \quad \leq 0 .
\end{aligned}
$$

Furthermore,

$$
\begin{aligned}
\left\langle(r f-A) z, y_{n}-z\right\rangle= & \left\langle(r f-A) z, y_{n}-x_{n}\right\rangle \\
& +\left\langle(r f-A) z, x_{n}-z\right\rangle .
\end{aligned}
$$

From (93) and (142), we have

$$
\lim _{n \rightarrow \infty} \sup \left\langle(r f-A) z, y_{n}-z\right\rangle \leq 0
$$

Step 5. Finally, we show that $x_{n}$ converges strongly to $z=$ $P_{\Theta}(I-A+r f) z$. Indeed, from (61) and (70), we obtain

$$
\begin{aligned}
& \left\|y_{n}-z\right\|^{2} \\
& =\| \varepsilon_{n} r f\left(x_{n}\right)+\beta_{n} x_{n} \\
& +\left(\left(1-\beta_{n}\right) I-\varepsilon_{n} A\right) z_{n}-z \|^{2} \\
& =\|\left(\left(1-\beta_{n}\right) I-\varepsilon_{n} A\right)\left(z_{n}-z\right) \\
& +\beta_{n}\left(x_{n}-z\right)+\varepsilon_{n}\left(r f\left(x_{n}\right)-A z\right) \|^{2} \\
& \leq\left\|\left(\left(1-\beta_{n}\right) I-\varepsilon_{n} A\right)\left(z_{n}-z\right)+\beta_{n}\left(x_{n}-p\right)\right\|^{2} \\
& +2 \varepsilon_{n}\left\langle\left(\left(1-\beta_{n}\right) I-\varepsilon_{n} A\right)\left(z_{n}-z\right)\right. \\
& +\beta_{n}\left(x_{n}-z\right)+\varepsilon_{n}\left(r f\left(x_{n}\right)-A z\right), \\
& \left.r f\left(x_{n}\right)-A p\right\rangle \\
& =\left\|\left(\left(1-\beta_{n}\right) I-\varepsilon_{n} A\right)\left(z_{n}-z\right)+\beta_{n}\left(x_{n}-z\right)\right\|^{2} \\
& +2 \varepsilon_{n}\left\langle\left(y_{n}-z, r f\left(x_{n}\right)-A z\right\rangle\right. \\
& \leq\left(1-\beta_{n}\right)\left\|\frac{\left(\left(1-\beta_{n}\right) I-\varepsilon_{n} A\right)}{1-\beta_{n}}\left(z_{n}-z\right)\right\|^{2} \\
& +\beta_{n}\left\|x_{n}-z\right\|^{2} \\
& +2 r \varepsilon_{n}\left\langle y_{n}-z, f\left(x_{n}\right)-f(z)\right\rangle \\
& +2 \varepsilon_{n}\left\langle y_{n}-z, f(z)-A z\right\rangle
\end{aligned}
$$




$$
\begin{aligned}
& \leq \frac{\left(1-\beta_{n}-\varepsilon_{n} \bar{\gamma}\right)^{2}}{1-\beta_{n}}\left\|x_{n}-z\right\|^{2} \\
& +\beta_{n}\left\|x_{n}-z\right\|^{2} \\
& +r \eta \varepsilon_{n}\left(\left\|x_{n}-z\right\|^{2}+\left\|y_{n}-z\right\|^{2}\right) \\
& +2 \varepsilon_{n}\left\langle y_{n}-z, f(z)-A z\right\rangle \\
& =\left(1-(2 \bar{\gamma}-r \eta)+\frac{\left(\varepsilon_{n} \bar{\gamma}\right)^{2}}{1-\beta_{n}}\right)\left\|x_{n}-z\right\|^{2} \\
& +r \eta \varepsilon_{n}\left\|y_{n}-z\right\|^{2} \\
& +2 \varepsilon_{n}\left\langle y_{n}-z, f(z)-A z\right\rangle
\end{aligned}
$$

which implies that

$$
\begin{aligned}
& \left\|y_{n}-z\right\|^{2} \\
& \leq\left(1-\frac{2(\bar{\gamma}-r \eta) \varepsilon_{n}}{1-r \eta \varepsilon_{n}}\right)\left\|x_{n}-z\right\|^{2} \\
& \quad+\frac{\varepsilon_{n}}{1-r \eta \varepsilon_{n}} \\
& \quad \times\left\{\frac{\bar{\gamma}^{2} \varepsilon_{n}}{1-\beta_{n}}\left\|x_{n}-z\right\|^{2}\right. \\
& \left.\quad+2\left\langle y_{n}-z, f(z)-A z\right\rangle\right\} .
\end{aligned}
$$

It follows from (146) that

$$
\begin{aligned}
& \left\|x_{n+1}-z\right\|^{2} \\
& =\left\|\gamma_{n} x_{n}+\left(1-\gamma_{n}\right) y_{n}-p\right\|^{2} \\
& \leq \gamma_{n}\left\|x_{n}-z\right\|^{2}+\left(1-\gamma_{n}\right)\left\|y_{n}-z\right\|^{2} \\
& \leq\left\|x_{n}-z\right\|^{2}+\left(1-\gamma_{n}\right) \\
& \times\left\{\left(1-\frac{2(\bar{\gamma}-r \eta) \varepsilon_{n}}{1-r \eta \varepsilon_{n}}\right)\left\|x_{n}-z\right\|^{2}\right. \\
& \quad+\frac{\varepsilon_{n}}{1-r \eta \varepsilon_{n}} \\
& \times\left(\frac{\bar{\gamma}^{2} \varepsilon_{n}}{1-\beta_{n}}\left\|x_{n}-z\right\|^{2}\right. \\
& \left.\left.+2\left\langle y_{n}-z, f(z)-A z\right\rangle\right)\right\}
\end{aligned}
$$

$$
\begin{aligned}
& =\left(1-\frac{2\left(1-\gamma_{n}\right)(\bar{\gamma}-r \eta) \varepsilon_{n}}{1-r \eta \varepsilon_{n}}\right)\left\|x_{n}-z\right\|^{2} \\
& +\frac{\left(1-\gamma_{n}\right) \varepsilon_{n}}{1-r \eta \varepsilon_{n}} \\
& \times\left(\frac{\bar{\gamma}^{2} \varepsilon_{n}}{1-\beta_{n}}\left\|x_{n}-z\right\|^{2}\right. \\
& \left.+2\left\langle y_{n}-z, f(z)-A z\right\rangle\right) .
\end{aligned}
$$

From condition (i) and (142), we know that

$$
\begin{gathered}
\sum_{i=1}^{\infty} \frac{2\left(1-\gamma_{n}\right)(\bar{\gamma}-r \eta) \varepsilon_{n}}{1-r \eta \varepsilon_{n}}=\infty, \\
\lim _{n \rightarrow \infty} \sup \frac{\left(1-\gamma_{n}\right) \varepsilon_{n}}{1-r \eta \varepsilon_{n}}\left(\frac{\bar{\gamma}^{2} \varepsilon_{n}}{1-\beta_{n}}\left\|x_{n}-z\right\|^{2}\right. \\
\left.+2\left\langle y_{n}-z, f(z)-A z\right\rangle\right) \leq 0 .
\end{gathered}
$$

we can conclude from Lemma 10 that $x_{n} \rightarrow z$ as $n \rightarrow \infty$. This completes the proof of Theorem 16.

Theorem 17. Let $C$ be a nonempty closed convex subset of a real Hilbert space $H$, and let $F_{k}, k \in\{1,2, \ldots, M\}$ be bifunction from $C \times C \rightarrow R$ satisfying (A1)-(A4). Let $\left\{T_{i}\right\}_{i=1}^{\infty}$ be $k_{i}$-strict pseudocontractive mappings of $C$ into self with $\kappa=\sup _{i} k_{i}$ and let $\rho_{j}=\left(\alpha_{1}^{j}, \alpha_{2}^{j}, \alpha_{2}^{j}\right) \in I \times I \times I$, where $I=[0,1]$, $\alpha_{1}^{j}+\alpha_{2}^{j}+\alpha_{2}^{j}=1, \alpha_{1}^{j}+\alpha_{2}^{j} \leq b<1$, and $\alpha_{1}^{j}, \alpha_{2}^{j}, \alpha_{2}^{j} \in(\kappa, 1)$ for all $j=1,2, \ldots$. For every $n \in N$, let $S_{n}$ and $S$ be $S$ mapping generated by $T_{n}, T_{n-1}, \ldots, T_{1}$ and $\rho_{n}, \rho_{n-1}, \ldots, \rho_{1}$ and $T_{n}, T_{n-1}, \ldots$, and $\rho_{n}, \rho_{n-1}, \ldots$, respectively. Let $B: C \rightarrow H$ be $\omega$-Lipschitz continuous and relaxed $(\mu, v)$-cocoercive mappings with $v-\mu \omega^{2}>0$, let $f$ be a contraction of $H$ into itself with $\eta \in(0,1)$, and let $A$ be a strongly positive linear bounded selfadjoint operator with the coefficients $\bar{\gamma}>0$ and $0<r<\bar{\gamma} / \eta$. Assume that

$$
\Theta:=\left(\bigcap_{i=1}^{\infty}\left\{F\left(T_{i}\right)\right\}\right) \cap\left(\bigcap_{k=1}^{M} \operatorname{SEP}\left(F_{k}\right)\right) \cap \operatorname{VI}(C, B) .
$$

Let $\left\{x_{n}\right\}$ be a sequence generated by $x_{1} \in C$ and

$$
\begin{gathered}
u_{n}=J_{r_{M, n}}^{F_{M}} J_{r_{M-1, n}}^{F_{M-1}} \cdots J_{r_{2, n}}^{F_{2}} J_{r_{1, n}}^{F_{1}} x_{n}, \\
z_{n}=P_{C}\left(S_{n} u_{n}-\alpha_{n} B S_{n} u_{n}\right), \\
y_{n}=\varepsilon_{n} r f\left(x_{n}\right)+\beta_{n} x_{n} \\
+\left(\left(1-\beta_{n}\right) I-\varepsilon_{n} A\right) z_{n}, \\
x_{n+1}=\gamma_{n} x_{n}+\left(1-\gamma_{n}\right) y_{n}, \quad \forall n \in N,
\end{gathered}
$$

where $\left\{S_{n}: C \rightarrow C\right\}$ is the sequence defined by (37). If $\left\{\varepsilon_{n}\right\}$, $\left\{\beta_{n}\right\}$ are two sequences in $(0,1)$ and $\left\{\gamma_{n}\right\} \subset\left[c_{1}, c_{2}\right] \subset(0,1)$ and 
$\left\{r_{k, n}\right\}$, for $k \in\{1,2, \ldots, M\}$ is a real sequence in $(0, \infty)$ satisfing the following conditions:

(i) $\lim _{n \rightarrow \infty} \varepsilon_{n}=0, \sum_{i=1}^{\infty} \varepsilon_{n}=\infty$,

(ii) $0<\lim _{n \rightarrow \infty} \inf \beta_{n} \leq \lim _{n \rightarrow \infty} \sup \beta_{n}<1$ and $\lim _{n \rightarrow \infty}\left|\beta_{n+1}-\beta_{n}\right|=0$,

(iii) $\lim _{n \rightarrow \infty} \inf r_{k, n}>0$ and $\lim _{n \rightarrow \infty}\left|r_{k, n+1}-r_{k, n}\right|=0$, for $k \in\{1,2, \ldots, M\}$,

(iv) $\left\{\alpha_{n}\right\} \subset\left[g_{1}, g_{2}\right] \subset\left(0,\left(2\left(v-\mu \omega^{2}\right)\right) / \omega^{2}\right)$ and $\lim _{n \rightarrow \infty}\left|\alpha_{n+1}-\alpha_{n}\right|=0$.

Then $\left\{x_{n}\right\}$ converges strongly to $z \in \Theta$, where $z$ is the unique solution of variational inequality

$$
\lim _{n \rightarrow \infty} \sup \langle(r f-A) z, p-z\rangle \leq 0, \quad \forall p \in \Theta,
$$

which is the optimality condition for the minimization problem

$$
\min _{z \in \Theta} \frac{1}{2}\langle A z, z\rangle-h(z)
$$

where $h$ is a potential function for $r f\left(\right.$ i.e., $h^{\prime}(z)=r f(z)$ for $z \in H)$.

Proof. By Theorem 16, for $i=1,2, \ldots, N$, letting $A_{i}=B$, we can obtain Theorem 17.

Theorem 18. Let $C$ be a nonempty closed convex subset of a real Hilbert space $H$, and let $F_{k}, k \in\{1,2, \ldots, M\}$ be a bifunction from $\mathrm{C} \times \mathrm{C} \rightarrow \mathrm{R}$ satisfying (A1)-(A4). Let $\left\{T_{i}\right\}_{i=1}^{\infty}$ be $k_{i}$-strict pseudo-contractive mappings of $C$ into self with $\kappa=\sup _{i} k_{i}$ and let $\rho_{j}=\left(\alpha_{1}^{j}, \alpha_{2}^{j}, \alpha_{2}^{j}\right) \in I \times I \times I$, where $I=[0,1]$, $\alpha_{1}^{j}+\alpha_{2}^{j}+\alpha_{2}^{j}=1, \alpha_{1}^{j}+\alpha_{2}^{j} \leq b<1$, and $\alpha_{1}^{j}, \alpha_{2}^{j}, \alpha_{2}^{j} \in(\kappa, 1)$ for all $j=1,2, \ldots$ For every $n \in N$, let $S_{n}$ and $S$ be $S$ mapping generated by $T_{n}, T_{n-1}, \ldots, T_{1}$ and $\rho_{n}, \rho_{n-1}, \ldots, \rho_{1}$ and $T_{n}, T_{n-1}, \ldots$, and $\rho_{n}, \rho_{n-1}, \cdots$, respectively. Let $A_{i}: C \rightarrow$ $H$ be $\omega_{i}$-Lipschitz continuous and relaxed $\left(\mu_{i}, v_{i}\right)$-cocoercive mappings with $v_{i}-\mu_{i} \omega_{i}^{2}>0$, for $i=1,2, \ldots, N$, let $f$ be a contraction of $H$ into itself with $\eta \in(0,1)$, and let $A$ be is a strongly positive linear bounded self-adjoint operator with the coefficients $\bar{\gamma}>0$ and $0<r<\bar{\gamma} / \eta$. Assume that

$$
\Theta:=\left(\bigcap_{i=1}^{\infty}\left\{F\left(T_{i}\right)\right\}\right) \cap \operatorname{EP}(F) \cap\left(\bigcap_{i=1}^{N} \operatorname{VI}\left(C, A_{i}\right)\right) .
$$

Let $\left\{x_{n}\right\}$ be a sequence generated by $x_{1} \in C$ and

$$
\begin{gathered}
F\left(u_{n}, y\right)+\frac{1}{r}\left(y-u_{n}, u_{n}-x_{n}\right) \geq 0, \\
z_{n}=P_{C}\left(S_{n} u_{n}-\alpha_{n}\left(\sum_{i=1}^{N} \delta_{i} A_{i}\right) S_{n} u_{n}\right), \\
y_{n}=\varepsilon_{n} r f\left(x_{n}\right)+\beta_{n} x_{n}+\left(\left(1-\beta_{n}\right) I-\varepsilon_{n} A\right) z_{n}, \\
x_{n+1}=\gamma_{n} x_{n}+\left(1-\gamma_{n}\right) y_{n}, \quad \forall n \in N,
\end{gathered}
$$

where $\left\{S_{n}: C \rightarrow C\right\}$ is the sequence defined by (37) and $\delta_{i} \epsilon$ $(0,1), \sum_{i=1}^{N} \delta_{i}=1$. If $\left\{\varepsilon_{n}\right\},\left\{\beta_{n}\right\}$ are two sequences in $(0,1)$ and $\left\{\gamma_{n}\right\} \subset\left[c_{1}, c_{2}\right] \subset(0,1)$ and $\left\{r_{k, n}\right\}$, for $k \in\{1,2, \ldots, M\}$ is a real sequence in $(0, \infty)$ satisfing the following conditions:

(i) $\lim _{n \rightarrow \infty} \varepsilon_{n}=0, \sum_{i=1}^{\infty} \varepsilon_{n}=\infty$,

(ii) $0<\lim _{n \rightarrow \infty} \inf \beta_{n} \leq \lim _{n \rightarrow \infty} \sup \beta_{n}<1$ and $\lim _{n \rightarrow \infty}\left|\beta_{n+1}-\beta_{n}\right|=0$,

(iii) $\lim _{n \rightarrow \infty}$ inf $r_{n}>0$ and $\lim _{n \rightarrow \infty}\left|r_{n+1}-r_{n}\right|=0$,

(iv) $\left\{\alpha_{n}\right\} \subset\left[g_{1}, g_{2}\right] \subset\left(0,\left(2 \sum_{i=1}^{N} \delta_{i}\left(v_{i}-\mu_{i} \omega_{i}^{2}\right)\right) /\left(\sum_{i=1}^{N} \delta_{i} \omega_{i}^{2}\right)\right)$ and $\lim _{n \rightarrow \infty}\left|\alpha_{n+1}-\alpha_{n}\right|=0$.

Then $\left\{x_{n}\right\}$ converges strongly to $z \in \Theta$, where $z$ is the unique solution of variational inequality

$$
\lim _{n \rightarrow \infty} \sup \langle(r f-A) z, p-z\rangle \leq 0, \quad \forall p \in \Theta,
$$

which is the optimality condition for the minimization problem

$$
\min _{z \in \Theta} \frac{1}{2}\langle A z, z\rangle-h(z)
$$

where $h$ is a potential function for $r f\left(\right.$ i.e., $h^{\prime}(z)=\operatorname{rf}(z)$ for $z \in H$ ).

Proof. By Theorem 16, letting $M=1$ for all $n \geq 1$, we can obtain Theorem 19.

Theorem 19. Let $C$ be a nonempty closed convex subset of a real Hilbert space $H$, and let $F_{k}, k \in\{1,2, \ldots, M\}$ be a bifunction from $C \times C \rightarrow R$ satisfying (A1)-(A4). Let $B: C \rightarrow H$ be $\omega$-Lipschitz continuous and relaxed $(\mu, v)$ cocoercive mappings with $v-\mu \omega^{2}>0$, and let $f$ be a contraction of $H$ into itself with $\eta \in(0,1)$, and let $A$ be is a strongly positive linear bounded self-adjoint operator with the coefficients $\bar{\gamma}>0$ and $0<r<\bar{\gamma} / \eta$. Assume that

$$
\Theta:=\left(\bigcap_{k=1}^{M} \operatorname{SEP}\left(F_{k}\right)\right) \cap \operatorname{VI}(C, B) .
$$

Let $\left\{x_{n}\right\}$ be a sequence generated by $x_{1} \in C$ and

$$
\begin{gathered}
u_{n}=J_{r_{M, n}}^{F_{M}} J_{r_{M-1, n}}^{F_{M-1}} \cdots J_{r_{2, n}}^{F_{2}} J_{r_{1, n}}^{F_{1}} x_{n}, \\
z_{n}=P_{C}\left(u_{n}-\alpha_{n} B u_{n}\right), \\
y_{n}=\varepsilon_{n} r f\left(x_{n}\right)+\beta_{n} x_{n}+\left(\left(1-\beta_{n}\right) I-\varepsilon_{n} A\right) z_{n}, \\
x_{n+1}=\gamma_{n} x_{n}+\left(1-\gamma_{n}\right) y_{n}, \quad \forall n \in N .
\end{gathered}
$$

If $\left\{\varepsilon_{n}\right\},\left\{\beta_{n}\right\}$ are two sequences in $(0,1)$ and $\left\{\gamma_{n}\right\} \subset\left[c_{1}, c_{2}\right] \subset$ $(0,1)$ and $\left\{r_{k, n}\right\}$, for $k \in\{1,2, \ldots, M\}$ is a real sequence in $(0, \infty)$ satisfing the following conditions:

(i) $\lim _{n \rightarrow \infty} \varepsilon_{n}=0, \sum_{i=1}^{\infty} \varepsilon_{n}=\infty$,

(ii) $0<\lim _{n \rightarrow \infty} \inf \beta_{n} \leq \lim _{n \rightarrow \infty} \sup \beta_{n}<1$ and $\lim _{n \rightarrow \infty}\left|\beta_{n+1}-\beta_{n}\right|=0$,

(iii) $\lim _{n \rightarrow \infty} \inf r_{k, n}>0$ and $\lim _{n \rightarrow \infty}\left|r_{k, n+1}-r_{k, n}\right|=0$, for $k \in\{1,2, \ldots, M\}$.

(iv) $\left\{\alpha_{n}\right\} \subset\left[g_{1}, g_{2}\right] \subset\left(0,\left(2\left(v-\mu \omega^{2}\right)\right) / \omega^{2}\right)$ and $\lim _{n \rightarrow \infty}\left|\alpha_{n+1}-\alpha_{n}\right|=0$. 
Then $\left\{x_{n}\right\}$ converges strongly to $z \in \Theta$, where $z$ is the unique solution of variational inequality

$$
\lim _{n \rightarrow \infty} \sup \langle(r f-A) z, p-z\rangle \leq 0, \quad \forall p \in \Theta,
$$

which is the optimality condition for the minimization problem

$$
\min _{z \in \Theta} \frac{1}{2}\langle A z, z\rangle-h(z)
$$

where $h$ is a potential function for $r f$ (i.e., $h^{\prime}(z)=r f(z)$ for $z \in H)$.

Proof. By Theorem 17, letting $T_{n}=I$ for all $n \leq 1$, we can obtain Theorem 19.

\section{Acknowledgments}

The authors would like to thank the anonymous referees and the editor for their constructive comments and suggestions, which greatly improved this paper. This project is supported by the Natural Science Foundation of China (Grant nos. 11171180, 11171193, 11126233, and 10901096) Shandong Provincial Natural Science Foundation (Grant no. ZR2011AM016), and the Project of Science and Technology Program of Weifang (Grant no. 20121103).

\section{References}

[1] E. Blum and W. Oettli, "From optimization and variational inequalities to equilibrium problems," The Mathematics Student, vol. 63, no. 1-4, pp. 123-145, 1994.

[2] A. Moudafi and M. Théra, "Proximal and dynamical approaches to equilibrium problems," in Ill-Posed Variational Problems and Regularization Techniques, vol. 477 of Lecture Notes in Economics and Mathematical Systems, pp. 187-201, Springer, Berlin, Germany, 1999.

[3] S. Plubtieng and P. Kumam, "Weak convergence theorem for monotone mappings and a countable family of nonexpansive mappings," Journal of Computational and Applied Mathematics, vol. 224, no. 2, pp. 614-621, 2009.

[4] V. Colao, G. Marino, and H. K. Xu, "An iterative method for finding common solutions of equilibrium and fixed point problems," Journal of Mathematical Analysis and Applications, vol. 344, no. 1, pp. 340-352, 2008.

[5] M. Tian, "A general iterative algorithm for nonexpansive mappings in Hilbert spaces," Nonlinear Analysis: Theory, Methods \& Applications, vol. 73, no. 3, pp. 689-694, 2010.

[6] Y. Yao, Y. C. Liou, and J. C. Yao, "Convergence theorem for equilibrium problems and fixed point problems of infinite family of nonexpansive mappings," Fixed Point Theory and Applications, vol. 2007, Article ID 064363, 12 pages, 2007.

[7] V. Colao and G. Marino, "Strong convergence for a minimization problem on points of equilibrium and common fixed points of an infinite family of nonexpansive mappings," Nonlinear Analysis: Theory, Methods \& Applications, vol. 73, no. 11, pp. 3513-3524, 2010.

[8] H. Iiduka and W. Takahashi, "Strong convergence theorems for nonexpansive mappings and inverse-strongly monotone mappings," Nonlinear Analysis: Theory, Methods \& Applications, vol. 61, no. 3, pp. 341-350, 2005.
[9] C. Jaiboon, P. Kumam, and U. W. Humphries, "Weak convergence theorem by an extragradient method for variational inequality, equilibrium and fixed point problems," Bulletin of the Malaysian Mathematical Sciences Society, vol. 32, no. 2, pp. 173185, 2009.

[10] P. Kumam and C. Jaiboon, "A system of generalized mixed equilibrium problems and fixed point problems for pseudocontractive mappings in Hilbert spaces," Fixed Point Theory and Applications, vol. 2010, Article ID 361512, 33 pages, 2010.

[11] T. Chamnarnpan and P. Kumam, "A new iterative method for a common solution of fixed points for pseudo-contractive mappings and variational inequalities," Fixed Point Theory and Applications, vol. 2012, article 67, 2012.

[12] T. Shimizu and W. Takahashi, "Strong convergence to common fixed points of families of nonexpansive mappings," Journal of Mathematical Analysis and Applications, vol. 211, no. 1, pp. 7183, 1997.

[13] K. K. Tan and H. K. Xu, "The nonlinear ergodic theorem for asymptotically nonexpansive mappings in Banach spaces," Proceedings of the American Mathematical Society, vol. 114, no. 2, pp. 399-404, 1992.

[14] T. Jitpeera and P. Kumam, "An extragradient type method for a system of equilibrium problems, variational inequality problems and fixed points of finitely many nonexpansive mappings," Journal of Nonlinear Analysis and Optimization: Theory and Applications, vol. 1, no. 1, pp. 71-91, 2010.

[15] P. Kumam and C. Jaiboon, "Approximation of common solutions to system of mixed equilibrium problems, variational inequality problem, and strict pseudo-contractive mappings," Fixed Point Theory and Applications, vol. 2011, Article ID 347204, 30 pages, 2011.

[16] G. Marino and H. K. Xu, "A general iterative method for nonexpansive mappings in Hilbert spaces," Journal of Mathematical Analysis and Applications, vol. 318, no. 1, pp. 43-52, 2006.

[17] P. Kumam and P. Katchang, "The hybrid algorithm for the system of mixed equilibrium problems, the general system of finite variational inequalities and common fixed points for nonexpansive semigroups and strictly pseudo-contractive mappings," Fixed Point Theory and Applications, vol. 2012, article 84, 2012.

[18] P. Kumam and P. Katchang, "A system of mixed equilibrium problems, a general system of variational inequality problems for relaxed cocoercive, and fixed point problems for nonexpansive semigroup and strictly pseudocontractive mappings," Journal of Applied Mathematics, vol. 2012, Article ID 414831, 35 pages, 2012.

[19] P. L. Combettes and S. A. Hirstoaga, "Equilibrium programming in Hilbert spaces," Journal of Nonlinear and Convex Analysis, vol. 6, no. 1, pp. 117-136, 2005.

[20] H. Zhou, "Convergence theorems of fixed points for $\kappa$-strict pseudo-contractions in Hilbert spaces," Nonlinear Analysis: Theory, Methods \& Applications, vol. 69, no. 2, pp. 456-462, 2008.

[21] F. E. Browder and W. V. Petryshyn, "Construction of fixed points of nonlinear mappings in Hilbert space," Journal of Mathematical Analysis and Applications, vol. 20, pp. 197-228, 1967.

[22] P. Kumam, U. Hamphries, and P. Katchang, "Common solutions of generalized mixed equilibrium problems, variational inclusions, and common fixed points for nonexpansive semigroups and strictly pseudocontractive mappings," Journal of Applied Mathematics, vol. 2011, Article ID 953903, 28 pages, 2011. 
[23] S. Takahashi and W. Takahashi, "Viscosity approximation methods for equilibrium problems and fixed point problems in Hilbert spaces," Journal of Mathematical Analysis and Applications, vol. 331, no. 1, pp. 506-515, 2007.

[24] Y. Liu, "A general iterative method for equilibrium problems and strict pseudo-contractions in Hilbert spaces," Nonlinear Analysis: Theory, Methods \& Applications, vol. 71, no. 10, pp. 4852-4861, 2009.

[25] K. Shimoji and W. Takahashi, "Strong convergence to common fixed points of infinite nonexpansive mappings and applications," Taiwanese Journal of Mathematics, vol. 5, no. 2, pp. 387404, 2001.

[26] K. Goebel and W. A. Kirk, Topics in Metric Fixed Point Theory, vol. 28 of Cambridge Studies in Advanced Mathematics, Cambridge University Press, Cambridge, UK, 1990.

[27] T. Suzuki, “Strong convergence of Krasnoselskii and Mann's type sequences for one-parameter nonexpansive semigroups without Bochner integrals," Journal of Mathematical Analysis and Applications, vol. 305, no. 1, pp. 227-239, 2005.

[28] H. K. Xu, "Viscosity approximation methods for nonexpansive mappings," Journal of Mathematical Analysis and Applications, vol. 298, no. 1, pp. 279-291, 2004.

[29] S. S. Chang, H. W. J. Lee, and C. K. Chan, "A new method for solving equilibrium problem fixed point problem and variational inequality problem with application to optimization," Nonlinear Analysis: Theory, Methods \& Applications, vol. 70, no. 9, pp. 3307-3319, 2009.

[30] H. H. Bauschke and J. M. Borwein, "On projection algorithms for solving convex feasibility problems," SIAM Review, vol. 38, no. 3, pp. 367-426, 1996.

[31] P. Combettes, "The foundations of set theoretic estimation," Proceedings of the IEEE, vol. 81, no. 2, pp. 182-208, 1993.

[32] W. Takahashi and M. Toyoda, "Weak convergence theorems for nonexpansive mappings and monotone mappings," Journal of Optimization Theory and Applications, vol. 118, no. 2, pp. 417428, 2003.

[33] I. Yamada, "The hybrid steepest descent method for the variational inequality problem over the intersection of fixed point sets of nonexpansive mappings," in Inherently Parallel Algorithm for Feasibility and Optimization, D. Butnariu, Y. Censor, and S. Reich, Eds., vol. 8 of Studies in Computational Mathematics, pp. 473-504, North-Holland, Amsterdam, The Netherlands, 2001.

[34] T. Chamnarnpan and P. Kumam, "Iterative algorithms for solving the system of mixed equilibrium problems, fixedpoint problems, and variational inclusions with application to minimization problem," Journal of Applied Mathematics, vol. 2012, Article ID 538912, 29 pages, 2012.

[35] A. Kangtunyakarn, "A new iterative scheme for fixed point problems of infinite family of $\kappa_{i}$-pseudo contractive mappings, equilibrium problem, variational inequality problems," Journal of Global Optimization, 2012.

[36] S. S. Chang, "Some problems and results in the study of nonlinear analysis," Nonlinear Analysis: Theory, Methods \& Applications, vol. 307, pp. 4197-4208, 1997.

[37] R. T. Rockafellar, "On the maximality of sums of nonlinear monotone operators," Transactions of the American Mathematical Society, vol. 149, pp. 75-88, 1970.

[38] A. Kangtunyakarn, "Strong convergence theorem for a generalized equilibrium problem and system of variational inequalities problem and infinite family of strict pseudo-contractions," Fixed Point Theory and Applications, vol. 2011, article 23, 2011. 


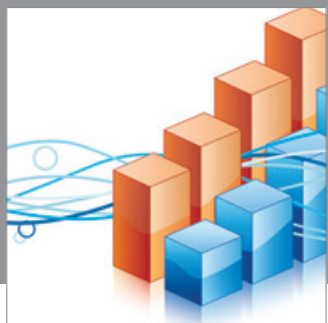

Advances in

Operations Research

mansans

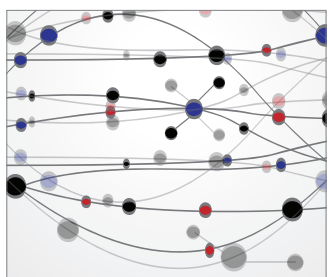

The Scientific World Journal
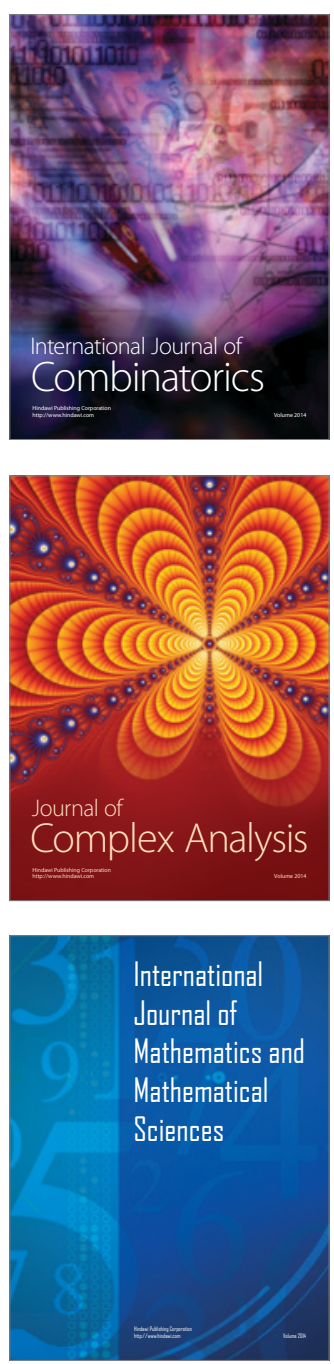
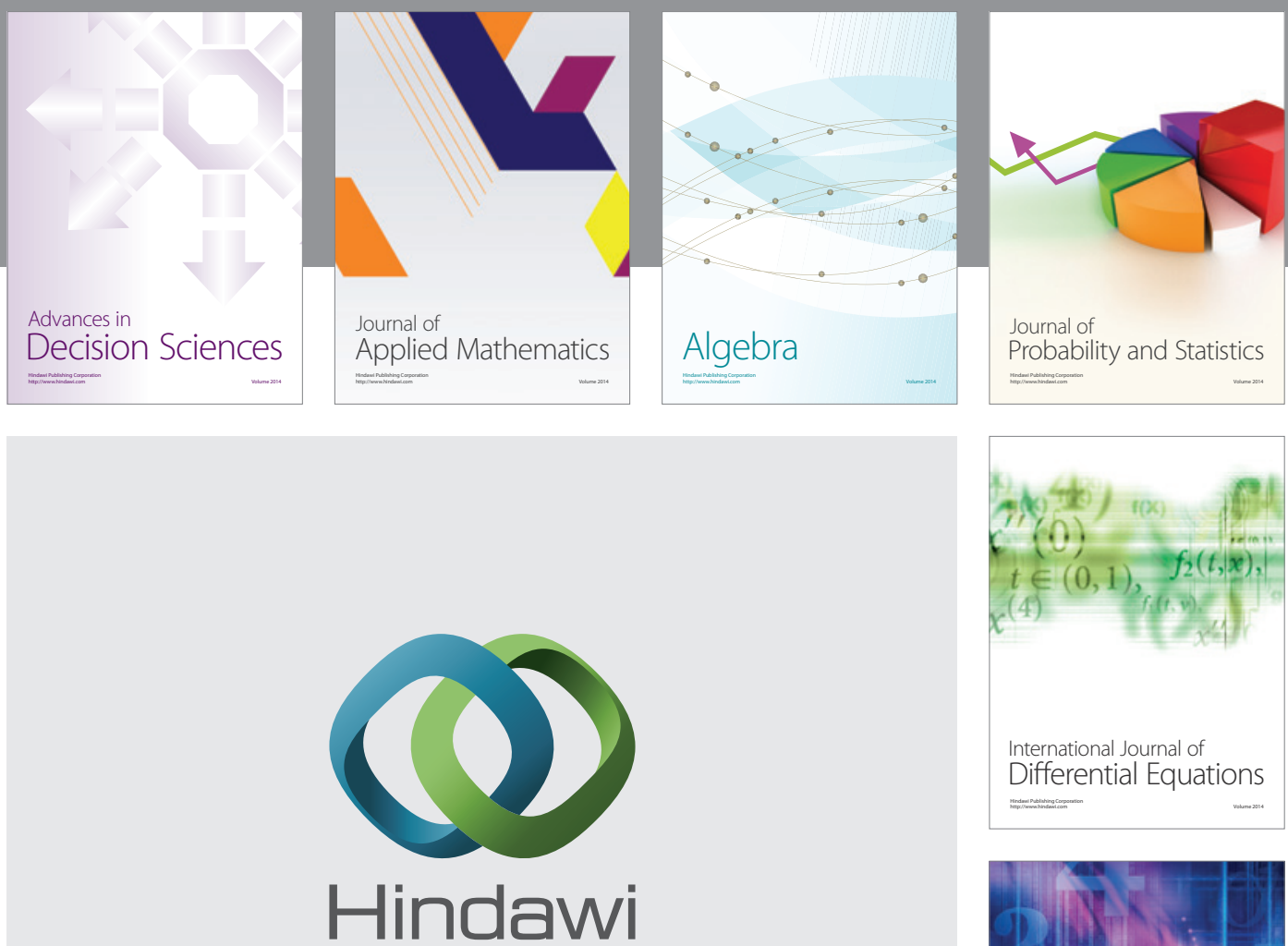

Submit your manuscripts at http://www.hindawi.com
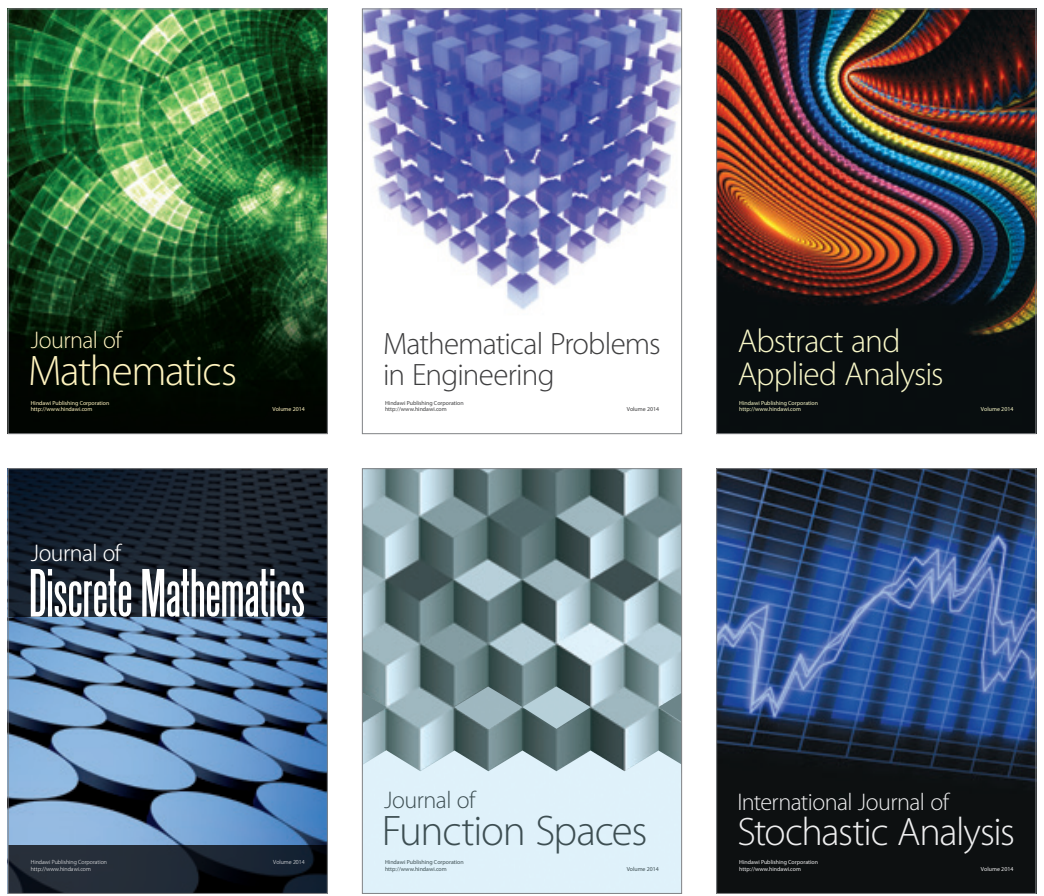

Journal of

Function Spaces

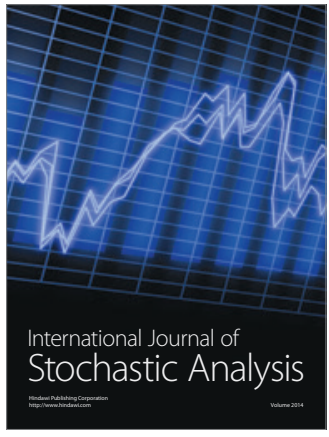

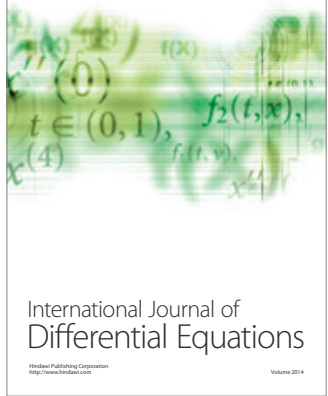
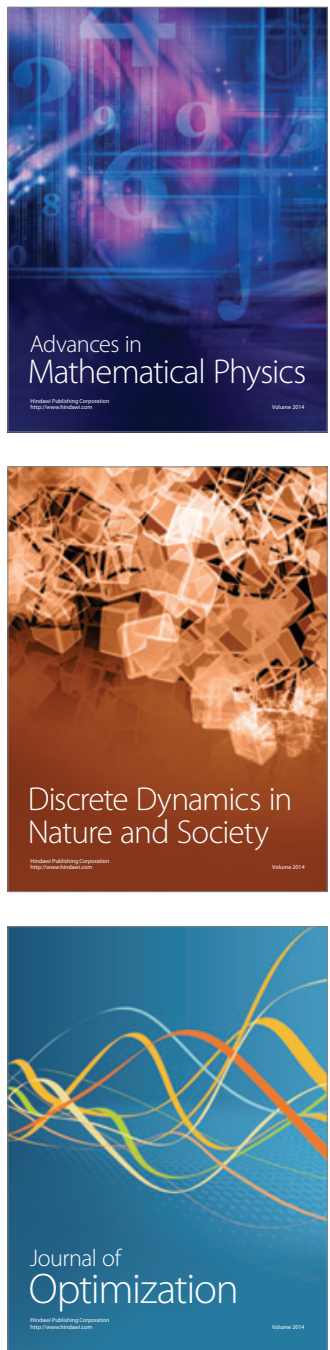